

\title{
Coleman maps and the $p$-adic regulator
}

\author{
Antonio Lei, David Loeffler and Sarah Livia Zerbes
}

We study the Coleman maps for a crystalline representation $V$ with non-negative Hodge-Tate weights via Perrin-Riou's $p$-adic "regulator" or "expanded logarithm" map $\mathscr{L}_{V}$. Denote by $\mathscr{H}(\Gamma)$ the algebra of $\mathbb{Q}_{p}$-valued distributions on $\Gamma=\operatorname{Gal}\left(\mathbb{Q}_{p}\left(\mu_{p^{\infty}}\right) / \mathbb{Q}_{p}\right)$. Our first result determines the $\mathscr{H}(\Gamma)$-elementary divisors of the quotient of $\mathbb{D}_{\text {cris }}(V) \otimes\left(\mathbb{B}_{\text {rig, } \mathbb{Q}_{p}}^{+}\right)^{\psi=0}$ by the $\mathscr{H}(\Gamma)$-submodule generated by $\left(\varphi^{*} \mathbb{N}(V)\right)^{\psi=0}$, where $\mathbb{N}(V)$ is the Wach module of $V$. By comparing the determinant of this map with that of $\mathscr{L}_{V}$ (which can be computed via PerrinRiou's explicit reciprocity law), we obtain a precise description of the images of the Coleman maps. In the case when $V$ arises from a modular form, we get some stronger results about the integral Coleman maps, and we can remove many technical assumptions that were required in our previous work in order to reformulate Kato's main conjecture in terms of cotorsion Selmer groups and bounded $p$-adic $L$-functions.

1. Introduction

1095

2. Refinements of crystalline representations and $\mathscr{H}(\Gamma)$-structure 1107

3. The construction of Coleman maps 1116

4. Images of the Coleman maps 1117

5. The Coleman maps for modular forms 1125

Acknowledgements 1129

References $\quad 1129$

\section{Introduction}

1A. Background. Let $p$ be an odd prime, and write $\mathbb{Q}_{\infty}=\mathbb{Q}\left(\mu_{p^{\infty}}\right)$. Define the Galois groups $\Gamma=\operatorname{Gal}\left(\mathbb{Q}_{\infty} / \mathbb{Q}\right)$ and $\Gamma_{1}=\operatorname{Gal}\left(\mathbb{Q}_{\infty} / \mathbb{Q}\left(\mu_{p}\right)\right)$. Note that $\Gamma \cong \Delta \times \Gamma_{1}$, where $\Delta$ is cyclic of order $p-1$ and $\Gamma_{1} \cong \mathbb{Z}_{p}$. For $H \in\left\{\Gamma, \Gamma_{1}\right\}$, denote by $\Lambda(H)$ the Iwasawa algebra of $H$, and $\Lambda_{\mathbb{Q}_{p}}(H)=\Lambda(H) \otimes_{\mathbb{Z}_{p}} \mathbb{Q}_{p}$.

The authors' research is supported by the following grants: ARC grant DP1092496 (Lei); EPSRC postdoctoral fellowship EP/F04304X/1 (Loeffler); EPSRC postdoctoral fellowship EP/F043007/1 (Zerbes).

MSC2010: primary 11R23; secondary 11F80, 11S25.

Keywords: $p$-adic regulator, Wach module, Selmer groups of modular forms. 
Let $V$ be a crystalline representation of $\mathscr{G}_{\mathbb{Q}_{p}}$ of dimension $d$ with non-negative Hodge-Tate weights. (We adopt the convention that the cyclotomic character has Hodge-Tate weight 1 , so this condition is equivalent to Fil $^{1} \mathbb{D}_{\text {cris }}(V)=0$.) We define

$$
H_{\mathrm{IW}}^{1}\left(\mathbb{Q}_{p}, V\right):=\mathbb{Q}_{p} \otimes_{\mathbb{Z}_{p}} \underset{\leftarrow}{\lim _{n}} H^{1}\left(\mathbb{Q}\left(\mu_{p^{n}}\right), T\right),
$$

where $T$ is a $\mathscr{G}_{\mathbb{Q}_{p}}$-stable $\mathbb{Z}_{p}$-lattice in $V$. This is a $\Lambda_{\mathbb{Q}_{p}}(\Gamma)$-module independent of the choice of $T$. In [Lei et al. 2010], we construct $\Lambda(\Gamma)$-homomorphisms (called the Coleman maps)

$$
\underline{\mathrm{Col}}_{i}: H_{\mathrm{Iw}}^{1}\left(\mathbb{Q}_{p}, V\right) \longrightarrow \Lambda_{\mathbb{Q}_{p}}(\Gamma)
$$

for $i=1, \ldots, d$, depending on a choice of basis of the Wach module $\mathbb{N}(V)$. In the case when $V=V_{f}(k-1)$, where $f=\sum a_{n} q^{n}$ is a modular eigenform of weight $k \geq 2$ and level coprime to $p$ (we assume that $a_{n} \in \mathbb{Q}$ for the time being in order to simplify notation) and $V_{f}$ is the 2-dimensional $p$-adic representation associated to $f$ by Deligne, these maps have two important applications. Firstly, we can define two $p$-adic $L$-functions $L_{p, 1}, L_{p, 2} \in \Lambda_{\mathbb{Q}_{p}}(\Gamma)$ on applying the Coleman maps to the localisation of the Kato zeta element as constructed in [Kato 2004]. In the supersingular case, i.e., when $p \mid a_{p}$, this enables us to obtain a decomposition of the $p$-adic $L$-functions defined in [Amice and Vélu 1975], which are not elements of $\Lambda_{\mathbb{Q}_{p}}(\Gamma)$ but of the distribution algebra $\mathscr{H}(\Gamma)$. More precisely, we show that there exists a $2 \times 2$ matrix $M \in M\left(2, \mathscr{H}\left(\Gamma_{1}\right)\right)$ depending only on $k$ and $a_{p}$ such that

$$
\left(\begin{array}{l}
L_{p, \alpha} \\
L_{p, \beta}
\end{array}\right)=M\left(\begin{array}{l}
L_{p, 1} \\
L_{p, 2}
\end{array}\right) .
$$

This generalises the results of [Pollack 2003] (when $a_{p}=0$ ) and [Sprung 2009] (when $f$ corresponds to an elliptic curve over $\mathbb{Q}$ and $p=3$ ). Secondly, by modifying the local conditions at $p$ in the definition of the $p$-Selmer group using the kernels of the maps $\underline{\mathrm{Col}}_{i}$, we define two new Selmer groups $\operatorname{Sel}_{p}^{i}\left(f / \mathbb{Q}_{\infty}\right)$. These are both $\Lambda(\Gamma)$-cotorsion, which is not true of the usual Selmer group in the supersingular case.

Fixing a character $\eta$ of $\Delta$ and restricting to the $\eta$-isotypical component, we get maps

$$
{\underline{\mathrm{Col}_{i}^{\eta}}}_{i}^{\eta}: H_{\mathrm{IW}}^{1}\left(\mathbb{Q}_{p}, V\right)^{\eta} \rightarrow \Lambda_{\mathbb{Q}_{p}}\left(\Gamma_{1}\right)
$$

Via the Poitou-Tate exact sequence, we can reformulate Kato's main conjecture (after tensoring with $\mathbb{Q}_{p}$ ) as follows:

Conjecture 1.1. For $i=1,2$, and each character $\eta$ of $\Delta$,

$$
\operatorname{Char}_{\Lambda_{\mathbb{Q}_{p}}\left(\Gamma_{1}\right)}\left(\mathbb{Q}_{p} \otimes_{\mathbb{Z}_{p}} \operatorname{Sel}_{p}^{i}\left(f / \mathbb{Q}_{\infty}\right)^{\eta, \vee}\right)=\operatorname{Char}_{\Lambda_{\mathbb{Q}_{p}}\left(\Gamma_{1}\right)}\left(\operatorname{Im}\left(\underline{\operatorname{Col}}_{i}^{\eta}\right) /\left(L_{p, i}^{\eta}\right)\right),
$$


where $M^{\vee}$ denotes the Pontryagin dual of a $\Lambda\left(\Gamma_{1}\right)$-module $M$ and $\operatorname{Char}_{\Lambda_{\mathbb{Q}_{p}}\left(\Gamma_{1}\right)} M$ denotes the $\Lambda_{\mathbb{Q}_{p}}\left(\Gamma_{1}\right)$-characteristic ideal of $M$.

When $v_{p}\left(a_{p}\right)$ is sufficiently large, we make use of the basis of $\mathbb{N}(V)$ constructed in [Berger et al. 2004] to show that the first Coleman map is surjective under some additional technical conditions. Therefore, we can rewrite Conjecture 1.1 as follows (see [Lei et al. 2010, Corollary 6.6]):

Theorem 1.2. Under certain technical conditions, the case $i=1$ in Conjecture 1.1 is equivalent to the assertion that $\operatorname{Char}_{\Lambda_{\mathbb{Q}_{p}}\left(\Gamma_{1}\right)}\left(\mathbb{Q}_{p} \otimes_{\mathbb{Z}_{p}} \operatorname{Sel}_{p}^{1}\left(f / \mathbb{Q}_{\infty}\right)^{\eta, \vee}\right)$ is generated by $L_{p, 1}^{\eta}$.

(In fact we can show that this equivalence holds integrally, i.e., without tensoring with $\left.\mathbb{Q}_{p}.\right)$

1B. Main results. In this paper, we extend the above results in several ways. Let $V$ be a crystalline representation of $\mathscr{G}_{\mathbb{Q}_{p}}$ of dimension $d$ with non-negative HodgeTate weights. We make the following assumption:

Assumption 1.3. The representation $V$ admits at least one non-critical refinement, after a suitable extension of coefficients.

See Section 1C5 below for the definition of a non-critical refinement. For now, let it suffice to say that this assumption holds for all 2-dimensional representations, and conjecturally for all representations "arising from geometry".

We identify $\Lambda\left(\Gamma_{1}\right)$ with the power series ring $\mathbb{Z}_{p} \llbracket X \rrbracket$, where $X=\gamma-1$ for a topological generator $\gamma$ of $\Gamma_{1}$. Denote by $\chi: \mathscr{G}_{\mathbb{Q}_{p}} \rightarrow \mathbb{Z}_{p}^{\times}$the cyclotomic character.

Firstly, we study the structure of $\mathbb{N}_{\text {rig }}(V):=\mathbb{N}(V) \otimes_{\mathbb{B}_{\mathbb{Q}_{p}}^{+}} \mathbb{B}_{\text {rig, } \mathbb{Q}_{p}}^{+}$as a $\Gamma$-module. If $\varphi^{*} \mathbb{N}_{\text {rig }}(V)$ denotes the $\mathbb{B}_{\text {rig, } \mathbb{Q}_{p}}^{+}$-span of $\varphi\left(\mathbb{N}_{\text {rig }}(V)\right)$, then $\left(\varphi^{*} \mathbb{N}_{\text {rig }}(V)\right)^{\psi=0}$ is contained in $\left(\mathbb{B}_{\text {rig, } \mathbb{Q}_{p}}^{+}\right)^{\psi=0} \otimes_{\mathbb{Q}_{p}} \mathbb{D}_{\text {cris }}(V)$, and both are free $\mathcal{H}(\Gamma)$-modules of rank equal to $d=\operatorname{dim}_{\mathbb{Q}_{p}} V$. We determine the elementary divisors of the quotient of these modules:

Theorem A (Theorem 2.10). The $\mathscr{H}(\Gamma)$-elementary divisors of the quotient

$$
\mathbb{D}_{\text {cris }}(V) \otimes_{\mathbb{Q}_{p}} \mathscr{H}(\Gamma) /\left(\varphi^{*} \mathbb{N}_{\text {rig }}(V)\right)^{\psi=0}
$$

are $\mathfrak{n}_{r_{1}}, \ldots, \mathfrak{n}_{r_{d}}$, where $r_{1}, \ldots, r_{d}$ are the Hodge-Tate weights of $V$ and

$$
\mathfrak{n}_{k}=\frac{\log (1+X)}{X} \cdots \frac{\log \left(\chi(\gamma)^{1-k}(1+X)\right)}{X-\chi(\gamma)^{k-1}+1} .
$$

This can be seen as a $\mathscr{H}(\Gamma)$-module analogue of [Berger 2004, Proposition III.2.1], which states that the $\mathbb{B}_{\text {rig, } \mathbb{Q}_{p}}^{+}$-elementary divisors of the quotient

$$
\left(\mathbb{B}_{\text {rig, } \mathbb{Q}_{p}}^{+} \otimes_{\mathbb{Q}_{p}} \mathbb{D}_{\text {cris }}(V)\right) / \mathbb{N}_{\text {rig }}(V)
$$


are $\left(\frac{t}{\pi}\right)^{r_{1}}, \ldots,\left(\frac{t}{\pi}\right)^{r_{d}}$. It is striking to note that for any $k \geq 0$, the Mellin transform of $\mathfrak{n}_{k}$ agrees with $(1+\pi) \varphi\left(\frac{t}{\pi}\right)^{k}$ up to a unit in $\mathbb{B}_{\text {rig, } \mathbb{Q}_{p}}^{+}$(see Proposition 1.6).

The second aim of this paper is to use Theorem A to determine the image of the map

$$
1-\varphi: \mathbb{N}_{\text {rig }}(V)^{\psi=1} \rightarrow\left(\varphi^{*} \mathbb{N}_{\text {rig }}(V)\right)^{\psi=0} .
$$

To do this, we make use of the following commutative diagram of $\mathscr{H}(\Gamma)$-modules:

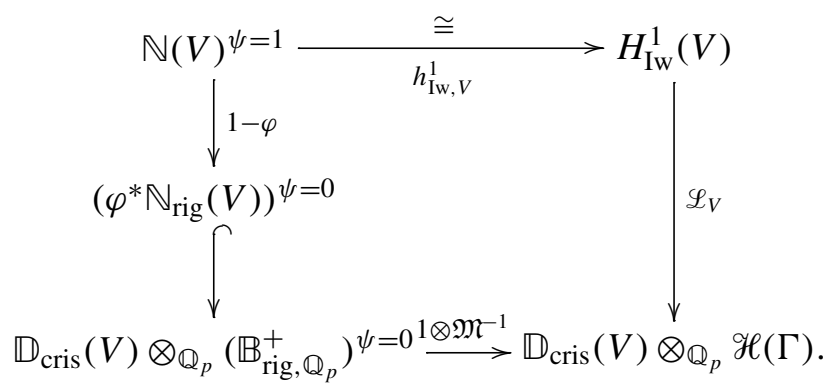

Here the map $\mathscr{L}_{V}$ is Perrin-Riou's "regulator" or "expanded logarithm" map (see [Perrin-Riou 1995]), which is a dual version of the more familiar exponential maps $\Omega_{V, h}$ appearing in [Perrin-Riou 1994]; and

$$
\mathfrak{M}: \mathscr{H}(\Gamma) \stackrel{\cong}{\longrightarrow}\left(\mathbb{B}_{\text {rig, }, \mathbb{Q}_{p}}^{+}\right)^{\psi=0}
$$

denotes the Mellin transform. The commutativity of the diagram is a theorem of Berger [2003, Theorem II.13]. Colmez's proof of the " $\delta_{V}$-conjecture" (see [Colmez 1998, Theorem IX.4.4]), which is part of Perrin-Riou's explicit reciprocity law, gives a formula for the determinant of the matrix of $\mathscr{L}_{V}$ (up to units). We can compare this with the determinant of the bottom left-hand map, which follows from Theorem A, to deduce that $1-\varphi: \mathbb{N}_{\text {rig }}(V)^{\psi=1} \rightarrow\left(\varphi^{*} \mathbb{N}_{\text {rig }}(V)\right)^{\psi=0}$ is surjective up to a small error term:

Theorem B (Corollary 4.13). Suppose that no eigenvalue of $\varphi$ on $\mathbb{D}_{\text {cris }}(V)$ lies in $p^{\mathbb{Z}}$. Then for each character $\eta$ of $\Delta$, there is a short exact sequence of $\mathscr{H}\left(\Gamma_{1}\right)$ modules

$$
0 \longrightarrow \mathbb{N}(V)^{\psi=1, \eta} \stackrel{1-\varphi}{\longrightarrow}\left(\varphi^{*} \mathbb{N}(V)\right)^{\psi=0, \eta} \stackrel{A_{\eta}}{\longrightarrow} \bigoplus_{i=0}^{r_{d}-1}\left(\mathbb{D}_{\text {cris }}(V) / V_{i, \eta}\right)\left(\chi^{i} \chi_{0}^{-i} \eta\right) \longrightarrow 0
$$

Here $V_{i, \eta}$ is a subspace of $\mathbb{D}_{\text {cris }}(V)$ of the same dimension as $\mathrm{Fil}^{-i} \mathbb{D}_{\text {cris }}(V)$, and the map $A_{\eta}$ is the composition of the inclusion of $\left(\varphi^{*} \mathbb{N}_{\text {rig }}(V)\right)^{\psi=0}$ in $\mathbb{D}_{\text {cris }}(V) \otimes_{\mathbb{Q}_{p}}$ $\mathscr{H}(\Gamma)$ with the map $\bigoplus_{i}\left(\mathrm{id} \otimes A_{\eta, i}\right)$, where $A_{\eta, i}$ is the natural reduction map $\mathscr{H}(\Gamma) \rightarrow$ $\mathbb{Q}_{p}\left(\chi^{i} \chi_{0}^{-i} \eta\right)$ obtained by quotienting out by the ideal $\left(X+1-\chi(\gamma)^{i}\right) \cdot e_{\eta}$. 
Using this we can describe the images of the Coleman maps (for any choice of basis of $\mathbb{N}(V))$ :

Theorem C (Corollary 4.15). Let $\eta$ be any character of $\Delta$. Then for all $1 \leq i \leq d$,

$$
\operatorname{Im}\left(\underline{\operatorname{Col}}_{i}^{\eta}\right)=\prod_{j \in I_{i}^{\eta}}\left(X-\chi(\gamma)^{j}+1\right) \Lambda_{\mathbb{Q}_{p}}\left(\Gamma_{1}\right)
$$

for some $I_{i}^{\eta} \subset\left\{0, \ldots, r_{d}-1\right\}$.

As a corollary of the proof, we also obtain a formula for the elementary divisors of the matrix of the map $\mathscr{L}_{V}$, which can be seen as a refinement of the statement of the $\delta(V)$-conjecture. For $i \in \mathbb{Z}$, define

$$
\ell_{i}=\frac{\log (1+X)}{\log _{p}(\chi(\gamma))}-i
$$

Theorem D (Theorem 4.16). The elementary divisors of the $\mathscr{H}(\Gamma)$-module quotient

$$
\frac{\mathscr{H}(\Gamma) \otimes_{\mathbb{Q}_{p}} \mathbb{D}_{\text {cris }}(V)}{\mathscr{H}(\Gamma) \otimes_{\Lambda_{\mathbb{Q}_{p}}(\Gamma)} \operatorname{Im}\left(\mathscr{L}_{V}\right)}
$$

are $\left[\lambda_{r_{1}} ; \lambda_{r_{2}} ; \ldots ; \lambda_{r_{d}}\right]$, where $\lambda_{k}=\ell_{0} \ell_{1} \ldots \ell_{k-1}$.

Suppose now that $V=V_{f}(k-1)$, where $f=\sum a_{n} e^{2 \pi i n z}$ is a modular form of weight $k \geq 2$ and level prime to $p$, and $V_{f}$ is the 2-dimensional $p$-adic representation associated to $f$ by Deligne. (Thus the Hodge-Tate weights of $V_{f}$ are 0 and $1-k$, and those of $V$ are 0 and $k-1$.) As we show in Section 1C5, Assumption 1.3 is automatically satisfied in this case, since $V$ is 2-dimensional. In this case, we can refine the results above to study the integral structure of the Coleman maps. Let $T_{f}$ be a $\mathscr{G}_{\mathbb{Q}_{p}}$-stable lattice in $V_{f}$, and let us assume that the $\mathbb{B}_{\mathbb{Q}_{p}}^{+}$-basis of $\mathbb{N}\left(V_{f}\right)$ used to define the Coleman maps is in fact an $\mathbb{A}_{\mathbb{Q}_{p}}^{+}$-basis of $\mathbb{N}\left(T_{f}\right)$.

Theorem $\mathbf{E}$ (Theorem 5.10). For $i=1,2$ and for each character $\eta$ of $\Delta$, the image of $H_{\mathrm{IW}}^{1}\left(\mathbb{Q}_{p}, T_{f}\right)^{\eta}$ under $\underline{\mathrm{Col}}_{i}^{\eta}$ is a submodule of finite index of the module

$$
\left(\prod_{j \in I_{i}^{\eta}}\left(X-\chi(\gamma)^{j}+1\right)\right) \Lambda\left(\Gamma_{1}\right)
$$

for some subset $I_{i}^{\eta} \subset\{0, \ldots, k-2\}$. Moreover, for each $\eta$ the sets $I_{1}^{\eta}$ and $I_{2}^{\eta}$ are disjoint.

This theorem generalises [Kurihara and Pollack 2007, Proposition 1.2], which determines the images of $\left(\underline{\mathrm{Col}}_{1}^{\Delta}, \underline{\mathrm{Col}}_{2}^{\Delta}\right)$ for elliptic curves with $a_{p}=0$. As a consequence of Theorem E, we can rewrite Conjecture 1.1 as below, without making any technical assumptions. 
Theorem F. For $i=1,2$, Conjecture 1.1 is equivalent to the assertion that for each $\eta$ the characteristic ideal $\operatorname{Char}_{\Lambda_{\mathbb{Q}_{p}}\left(\Gamma_{1}\right)}\left(\mathbb{Q}_{p} \otimes_{\mathbb{Z}_{p}} \operatorname{Sel}_{p}^{i}\left(f / \mathbb{Q}_{\infty}\right)^{\eta, \vee}\right)$ is generated by $L_{p, i}^{\eta} / \prod_{j \in I_{i}^{\eta}}\left(X-\chi(\gamma)^{j}+1\right)$ where $I_{i}^{\eta}$ is as given by Theorem $E$.

Finally, we explain in Section $5 \mathrm{C}$ how it is possible to choose a basis in such a way that $I_{1}^{\eta}=I_{2}^{\eta}=\varnothing$, i.e., the modules $\Lambda\left(\Gamma_{1}\right) / \operatorname{Im}\left(\underline{\mathrm{Col}}_{i}^{\eta}\right)$ are pseudo-null for both $i=1$ and 2 .

Remark 1.4. The local results in this paper (Theorems A, B, C and D) hold with representations of $\mathscr{G}_{\mathbb{Q}_{p}}$ replaced by representations of $\mathscr{G}_{F}$ for an arbitrary finite unramified extension $F / \mathbb{Q}_{p}$, with essentially the same proofs. We have chosen to work over $\mathbb{Q}_{p}$ for the sake of simplicity, since this is all that is needed for applications to modular forms.

In [Loeffler and Zerbes 2012], these methods are applied to the study of the "critical-slope" $L$-function attached to an ordinary modular form (corresponding to the non-unit Frobenius eigenvalue).

\section{C. Setup and notation.}

1C1. Fontaine rings. We review the definitions of the Fontaine rings we use in this paper. Details can be found in [Berger 2004] or [Lei et al. 2010].

Throughout this paper, $p$ is an odd prime. If $K$ is a number field or a local field of characteristic 0 , then $G_{K}$ denotes its absolute Galois group and $O_{K}$ the ring of integers of $K$. We write $\Gamma$ for the Galois group $\operatorname{Gal}\left(\mathbb{Q}\left(\mu_{p^{\infty}}\right) / \mathbb{Q}\right)$, which we identify with $\mathbb{Z}_{p}^{\times}$via the cyclotomic character $\chi$. Then $\Gamma \cong \Delta \times \Gamma_{1}$, where $\Delta$ is of order $p-1$ and $\Gamma_{1} \cong \mathbb{Z}_{p}$. We fix a topological generator $\gamma$ of $\Gamma_{1}$.

We write $\mathbb{B}_{\text {rig, } \mathbb{Q}_{p}}^{+}$for the ring of power series $f(\pi) \in \mathbb{Q}_{p} \llbracket \pi \rrbracket$ such that $f(X)$ converges everywhere on the open unit $p$-adic disc. Equip $\mathbb{B}_{\text {rig, } \mathbb{Q}_{p}}^{+}$with actions of $\Gamma$ and a Frobenius operator $\varphi$ by $g . \pi=(\pi+1)^{\chi(g)}-1$ and $\varphi(\pi)=(\pi+1)^{p}-1$. We can then define a left inverse $\psi$ of $\varphi$ satisfying

$$
\varphi \circ \psi(f(\pi))=\frac{1}{p} \sum_{\zeta^{p}=1} f(\zeta(1+\pi)-1) .
$$

Inside $\mathbb{B}_{\text {rig, } \mathbb{Q}_{p}}^{+}$, we have subrings $\mathbb{A}_{\mathbb{Q}_{p}}^{+}=\mathbb{Z}_{p} \llbracket \pi \rrbracket$ and $\mathbb{B}_{\mathbb{Q}_{p}}^{+}=\mathbb{Q}_{p} \otimes_{\mathbb{Z}_{p}} \mathbb{A}_{\mathbb{Q}_{p}}^{+}$. Moreover, the actions of $\varphi, \psi$ and $\Gamma$ preserve these subrings. Finally, we write $t=$ $\log (1+\pi) \in \mathbb{B}_{\mathrm{rig}, \mathbb{Q}_{p}}^{+}$and $q=\varphi(\pi) / \pi \in \mathbb{A}_{\mathbb{Q}_{p}}^{+}$. A formal power series calculation shows that $g(t)=\chi(g) t$ for $g \in \Gamma$ and $\varphi(t)=p t$.

1C2. Iwasawa algebras and power series. Given a finite extension $K$ of $\mathbb{Q}_{p}$, denote by $\Lambda_{\mathscr{O}_{K}}(\Gamma)$ (respectively $\Lambda_{\mathscr{O}_{K}}\left(\Gamma_{1}\right)$ ) the Iwasawa algebra $\mathbb{Z}_{p} \llbracket \Gamma \rrbracket \otimes_{\mathbb{Z}_{p}} \boldsymbol{O}_{K}$ (respectively $\mathbb{Z}_{p} \llbracket\left[\Gamma_{1} \rrbracket \otimes_{\mathbb{Z}_{p}} \widehat{O}_{K}\right)$ over $\mathscr{O}_{K}$. We further write $\Lambda_{K}(\Gamma)=\mathbb{Q} \otimes \Lambda_{\mathscr{C}_{K}}(\Gamma)$ and $\Lambda_{K}\left(\Gamma_{1}\right)=\mathbb{Q} \otimes \Lambda_{\mathscr{O}_{K}}\left(\Gamma_{1}\right)$. If $M$ is a finitely generated torsion $\Lambda_{\mathscr{O}_{K}}\left(\Gamma_{1}\right)$-module, we write $\operatorname{Char}_{\Lambda_{\emptyset_{K}}\left(\Gamma_{1}\right)}(M)$ for its characteristic ideal. 
Let $\mathscr{H}(\Gamma)$ be the space of distributions on $\Gamma$ (the continuous dual of the space of locally analytic functions on $\Gamma$ ), with the ring structure defined by convolution. We may identify this with the space of formal power series

$$
\left\{f \in \mathbb{Q}_{p}[\Delta] \llbracket X \rrbracket: f \text { converges everywhere on the open unit } p \text {-adic disc }\right\},
$$

where $X$ corresponds to $\gamma-1$. We may identify $\Lambda_{\mathbb{Q}_{p}}(\Gamma)$ with the subring of $\mathscr{H}(\Gamma)$ consisting of power series with bounded coefficients.

The action of $\Gamma$ on $\mathbb{B}_{\text {rig, } \mathbb{Q}_{p}}^{+}$gives an isomorphism of $\mathscr{H}(\Gamma)$ with $\left(\mathbb{B}_{\text {rig, } \mathbb{Q}_{p}}^{+}\right)^{\psi=0}$, the Mellin transform

$$
\begin{aligned}
\mathfrak{M}: \mathscr{H}(\Gamma) & \rightarrow\left(\mathbb{B}_{\text {rig, } \mathbb{Q}_{p}}^{+}\right)^{\psi=0} \\
f(\gamma-1) & \mapsto f(\gamma-1) \cdot(\pi+1) .
\end{aligned}
$$

In particular, $\Lambda_{\mathbb{Z}_{p}}(\Gamma)$ corresponds to $\left(\mathbb{A}_{\mathbb{Q}_{p}}^{+}\right)^{\psi=0}$ under $\mathfrak{M}$. Similarly, we define $\mathscr{H}\left(\Gamma_{1}\right)$ as the subring of $\mathscr{H}(\Gamma)$ defined by power series over $\mathbb{Q}_{p}$, rather than $\mathbb{Q}_{p}[\Delta]$. Then, $\mathscr{H}\left(\Gamma_{1}\right)$ corresponds to $(1+\pi) \varphi\left(\mathbb{B}_{\text {rig, } \mathbb{Q}_{p}}^{+}\right)$under $\mathfrak{M}$, and $\Lambda_{\mathbb{Z}_{p}}\left(\Gamma_{1}\right)$ to $(1+$ $\pi) \varphi\left(\mathbb{A}_{\mathbb{Q}_{p}}^{+}\right)$. (See [Perrin-Riou 2001, B.2.8] for more details.)

If $d$ is an integer and $S$ is a $\Lambda_{K}\left(\Gamma_{1}\right)$-submodule of $K \otimes_{\mathbb{Q}_{p}} \mathscr{H}\left(\Gamma_{1}\right)^{\oplus d}$ which is free of rank $d$, we write $\operatorname{det}(S)$ for the determinant of any basis of $S$, which is well-defined up to multiplication by a unit of $\Lambda_{K}\left(\Gamma_{1}\right)$. If $F$ is a homomorphism of $\Lambda_{K}\left(\Gamma_{1}\right)$-modules whose image is a free rank $d \Lambda_{K}\left(\Gamma_{1}\right)$-submodule of $K \otimes_{\mathbb{Q}_{p}}$ $\mathscr{H}\left(\Gamma_{1}\right)^{\oplus d}$, we write $\operatorname{det}(F)$ for $\operatorname{det}(\operatorname{Im}(F))$.

For an integer $i$, define

$$
\left.\begin{array}{l}
\ell_{i}=\frac{\log (1+X)}{\log _{p}(\chi(\gamma))}-i \\
\delta_{i}=\frac{\ell_{i}}{X+1-\chi(\gamma)^{i}}
\end{array}\right\} \in \mathscr{H}\left(\Gamma_{1}\right) .
$$

Note that $\ell_{i}$ is independent of the choice of generator $\gamma$ (hence the choice of normalising factor), but $\delta_{i}$ is not.

Remark 1.5. Note that for any positive integer $k$, we have

$$
\mathfrak{n}_{k}=a_{k} \delta_{k-1} \ldots \delta_{0}
$$

where $a_{k}=\log (\chi(\gamma))^{k} \in \mathbb{Z}_{p}$ is nonzero.

The following result slightly refines [Berger 2003, Lemma II.2]. 
Proposition 1.6. For any $k \geq 0$, we have

$$
\begin{aligned}
& \mathfrak{M}\left(\ell_{k-1} \ldots \ell_{0} \mathscr{H}(\Gamma)\right)=\left(t^{k} \mathbb{B}_{\text {rig, } \mathbb{Q}_{p}}^{+}\right)^{\psi=0} \\
& \mathfrak{M}\left(\delta_{k-1} \ldots \delta_{0} \mathscr{H}(\Gamma)\right)=\left(\left(\frac{t}{\varphi(\pi)}\right)^{k} \mathbb{B}_{\text {rig, } \mathbb{Q}_{p}}^{+}\right)^{\psi=0} .
\end{aligned}
$$

Proof. One checks easily that $\ell_{i}$ acts on $\mathbb{B}_{\text {rig, } \mathbb{Q}_{p}}^{+}$as the differential operator

$$
(1+\pi) t \frac{\mathrm{d}}{\mathrm{d} \pi}-i
$$

and hence

$$
\ell_{j}\left(t^{j} f\right)=t^{j+1}(1+\pi) \frac{\mathrm{d} f}{\mathrm{~d} \pi} .
$$

Since $(1+\pi) \frac{\mathrm{d}}{\mathrm{d} \pi}$ is an isomorphism on $\left(\mathbb{B}_{\text {rig, } \mathbb{Q}_{p}}^{+}\right)^{\psi=0}$ (it is the map on distributions dual to the map $f(x) \mapsto x f(x)$ on functions), it follows that each $\ell_{j}$ maps $\left(t^{j} \mathbb{B}_{\mathrm{rig}, \mathbb{Q}_{p}}^{+}\right)^{\psi=0}$ bijectively onto $\left(t^{j+1} \mathbb{B}_{\mathrm{rig}, \mathbb{Q}_{p}}^{+}\right)^{\psi=0}$.

To prove a similar statement for the $\delta_{i}$, we note that

$$
\left(\mathbb{B}_{\text {rig, } \mathbb{Q}_{p}}^{+} / \varphi(\pi) \mathbb{B}_{\text {rig, } \mathbb{Q}_{p}}^{+}\right)^{\psi=0}
$$

is isomorphic to $\mathbb{Q}_{p}[\Delta]$ as a $\Gamma$-module. Since $t$ is a uniformiser of the ideal $\varphi(\pi)$, we have

$$
\left(\varphi(\pi)^{j} \mathbb{B}_{\text {rig, } \mathbb{Q}_{p}}^{+} / \varphi(\pi)^{j+1} \mathbb{B}_{\text {rig, }, \mathbb{Q}_{p}}^{+}\right)^{\psi=0}=\left(t^{j} \mathbb{B}_{\text {rig, } \mathbb{Q}_{p}}^{+} / t^{j} \varphi(\pi) \mathbb{B}_{\text {rig, } \mathbb{Q}_{p}}^{+}\right)^{\psi=0} \cong \mathbb{Q}_{p}[\Delta](j)
$$

as a $\Gamma$-module. Hence its annihilator is $X+1-\chi(\gamma)^{j}$. These factors are mutually coprime and coprime to $\delta_{0} \ldots \delta_{k-1}$, and the product is $\ell_{0} \ldots \ell_{k-1}$, so the result follows.

1C3. Isotypical components. Let $\eta: \Delta \rightarrow \mathbb{Z}_{p}^{\times}$be a character. We write $e_{\eta}=$ $(p-1)^{-1} \sum_{\sigma \in \Delta} \eta^{-1}(\sigma) \sigma$. If $M$ is a $\Lambda_{E}(\Gamma)$-module, its $\eta$-isotypical component is given by $M^{\eta}=e_{\eta} M$. When $\eta=1$, we write $M^{\Delta}$ in place of $M^{\eta}$.

We identify $\Lambda_{E}\left(\Gamma_{1}\right)$ with the power series in $X=\gamma-1$ with bounded coefficients in $E$. Given

$$
F=\sum_{\sigma \in \Delta, n \geq 0} a_{\sigma, n} \sigma(\gamma-1)^{n} \in \Lambda(\Gamma),
$$

we write $F^{\eta}=e_{\eta} F$ for its image in $\Lambda_{E}(\Gamma)^{\eta}$. In particular,

$$
F^{\eta}=e_{\eta} \sum_{n \geq 0}\left(\sum_{\sigma \in \Delta} a_{\sigma, n} \eta(\sigma)\right)(\gamma-1)^{n} \in e_{\eta} \Lambda_{E}\left(\Gamma_{1}\right) .
$$

Therefore, we can identify $F^{\eta}$ with a power series in $X=\gamma-1$. Under this identification, the value $\left.F^{\eta}\right|_{X=\chi(\gamma)^{j}-1}$ is given by $\chi^{j} \chi_{0}^{-j} \eta(F)$ where $\chi_{0}=\left.\chi\right|_{\Delta}$ for all $j \in \mathbb{Z}$. 
1C4. Crystalline representations. Let $E$ and $F$ be finite extensions of $\mathbb{Q}_{p}$. Let $V$ be a crystalline $E$-linear representation of $G_{\mathbb{Q}_{p}}$. We denote the Dieudonné module of $V$ by $\mathbb{D}_{\text {cris }}(V)$. If $j \in \mathbb{Z}, \mathrm{Fil}^{j} \mathbb{D}_{\text {cris }}(V)$ denotes the $j$ th step in the de Rham filtration of $\mathbb{D}_{\text {cris }}(V)$. We say $V$ is positive if $\mathbb{D}_{\text {cris }}(V)=$ Fil $^{0} \mathbb{D}_{\text {cris }}(V)$ (following the standard, but unfortunate, convention that positive representations are precisely those with non-positive Hodge-Tate weights).

The $(\varphi, \Gamma)$-module of $V$ is denoted by $\mathbb{D}(V)$. As shown by Fontaine (unpublished; for a reference see [Cherbonnier and Colmez 1999, Section II]), we have a canonical isomorphism of $\Lambda_{E}(\Gamma)$-modules

$$
h_{\mathrm{Iw}, V}^{1}: \mathbb{D}(V)^{\psi=1} \rightarrow H_{\mathrm{Iw}}^{1}\left(\mathbb{Q}_{p}, V\right) .
$$

We write $\exp _{F, V}: F \otimes \mathbb{D}_{\text {cris }}(V) \rightarrow H^{1}(F, V)$ for the Bloch-Kato exponential over $F$.

For an integer $j, V(j)$ denotes the $j$ th Tate twist of $V$, i.e., $V(j)=V \otimes E e_{j}$ where $G_{\mathbb{Q}_{p}}$ acts on $e_{j}$ via $\chi^{j}$. We have

$$
\mathbb{D}_{\text {cris }}(V(j))=t^{-j} \mathbb{D}_{\text {cris }}(V) \otimes e_{j}
$$

For any $v \in \mathbb{D}_{\text {cris }}(V), v_{j}=t^{-j} v \otimes e_{j}$ denotes its image in $\mathbb{D}_{\text {cris }}(V(j))$.

If $h \geq 1$ is an integer such that $\operatorname{Fil}^{-h} \mathbb{D}_{\text {cris }}(V)=\mathbb{D}_{\text {cris }}(V)$, we write $\Omega_{V, h}$ for the Perrin-Riou exponential as defined in [Perrin-Riou 1994].

Let $T$ be an $O_{E}$-lattice in $V$ which is stable under $G_{\mathbb{Q}_{p}}$. We denote the Wach module of $V$ (respectively $T$ ) by $\mathbb{N}(V)$ (respectively $\mathbb{N}(T)$ ), a free module of rank $d$ over $\mathbb{B}_{\mathbb{Q}_{p}}^{+}$(respectively $\left.\mathbb{A}_{\mathbb{Q}_{p}}^{+}\right)$. Recall that $\Gamma$ acts on both of these objects, and there is a map $\varphi: \mathbb{N}(T)\left[\pi^{-1}\right] \rightarrow \mathbb{N}(T)\left[\varphi(\pi)^{-1}\right]$, preserving $\mathbb{N}(T)$ if $T$ is positive (and similarly for $V$ ).

For any $j \in \mathbb{Z}$ we can identify $\mathbb{N}(T(j))$ with $\pi^{-j} \mathbb{N}(T) \otimes e_{j}$, where $e_{j}$ is as above. Given an $R$-module $M$ with an action of $\varphi$ and a submodule $N, \varphi^{*} N$ denotes the $R$-submodule of $M$ generated by $\varphi(N)$, e.g., $\varphi^{*} \mathbb{N}(T)$ denotes the $\mathbb{A}_{\mathbb{Q}_{p}}^{+}$-submodule of $\mathbb{N}(T)\left[\pi^{-1}\right]$ generated by $\varphi(\mathbb{N}(T))$. Finally, we write

$$
\mathbb{N}_{\text {rig }}(V)=\mathbb{N}(V) \otimes_{\mathbb{B}_{\mathbb{Q}_{p}}^{+}} \mathbb{B}_{\text {rig, } \mathbb{Q}_{p}}^{+} .
$$

The following lemma is implicit in the calculations of [Lei et al. 2010, §3], but for the convenience of the reader we give a separate proof:

Lemma 1.7. If the Hodge-Tate weights of $V$ are $\geq 0$, then we have

$$
\mathbb{N}(T) \subseteq \varphi^{*} \mathbb{N}(T)
$$

and similarly for $\mathbb{N}(V)$.

Proof. It suffices to prove the result for $T$. Suppose that the Hodge-Tate weights of $V$ are in $[0, m]$. Then $\mathbb{N}(T)=\pi^{-m} \mathbb{N}(T(-m))$. Since $T(-m)$ is positive, $\varphi$ 
preserves $\mathbb{N}(T(-m))$ and $\mathbb{N}(T(-m)) / \varphi^{*} \mathbb{N}(T(-m))$ is killed by $q^{m}$ [Berger 2004, proof of Theorem III.3.1]. Equivalently, we have

$$
q^{m} \cdot \pi^{m} \mathbb{N}(T) \subseteq \varphi^{*}\left(\pi^{m} \mathbb{N}(T)\right)=\varphi(\pi)^{m} \varphi^{*} \mathbb{N}(T) .
$$

Since $q=\varphi(\pi) / \pi$, the result follows.

1C5. Refinements of crystalline representations. Let $V$ be an $E$-linear crystalline representation of $G_{\mathbb{Q}_{p}}$ of dimension $d$, and let $s_{1} \leq \cdots \leq s_{d}$ be the jumps in the filtration of $\mathbb{D}_{\text {cris }}(V)$, so the Hodge-Tate weights are $-s_{i}$. If $Y$ is an $E$-linear subspace of $\mathbb{D}_{\text {cris }}(V)$ of dimension $e \leq d$, we say $Y$ is in general position (with respect to the Hodge filtration) if the intersections $\mathrm{Fil}^{j} Y=Y \cap \mathrm{Fil}^{j} \mathbb{D}_{\text {cris }}(V)$ have the smallest possible dimension; that is,

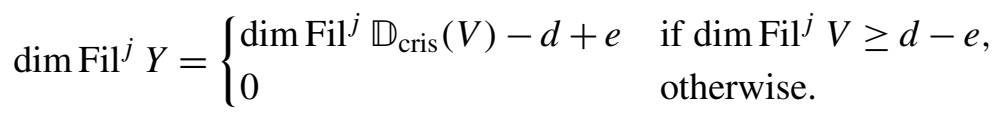

This is equivalent to the requirement that the jumps of the filtration $\mathrm{Fil}^{j} Y$ are $s_{1}, \ldots, s_{e}$.

As in [Bellaïche and Chenevier 2009, §2.4.1], we define a refinement of $V$ to be a family $\underline{Y}=\left(Y_{i}\right)_{i=1}^{d}$ of $E$-linear subspaces of $\mathbb{D}_{\text {cris }}(V)$ stable under $\varphi$, with $0 \subsetneq Y_{1} \subsetneq \cdots \subsetneq Y_{d}=\mathbb{D}_{\text {cris }}(V)$, so $\operatorname{dim}_{E} Y_{i}=i$. It is clear that refinements exist if and only if the eigenvalues of $\varphi$ on $\mathbb{D}_{\text {cris }}(V)$ lie in $E$.

We say that the refinement is non-critical if each of the subspaces $Y_{i}$ is in general position, or equivalently if $Y_{i} \cap \mathrm{Fil}^{s_{i}+1} \mathbb{D}_{\text {cris }}(V)=0$ for all $i$.

(If the Hodge-Tate weights of $V$ are distinct, as Bellaïche and Chenevier assume, then this is equivalent to the assertion that $\mathbb{D}_{\text {cris }}(V)=Y_{i} \oplus \mathrm{Fil}^{s_{i}+1} \mathbb{D}_{\text {cris }}(V)$ for each $i$, which coincides with Definition 2.4.5 of [op. cit.]).

Proposition 1.8. If the eigenvalues of Frobenius on $\mathbb{D}_{\text {cris }}(V)$ lie in $E$, and either $d=2$ or $\varphi$ acts semisimply on $\mathbb{D}_{\text {cris }}(V)$, then there exists a non-critical refinement of $V$.

Proof. As noted in [Bellaïche and Chenevier 2009, Remark 2.4.6(iii)], the case where $\varphi$ acts semisimply is obvious: any basis of eigenvectors of $\mathbb{D}_{\text {cris }}(V)$ defines $d$ ! refinements, one for each ordering of the basis vectors, and it is easy to see that we can choose an ordering such that the resulting refinement is non-critical. Hence let us assume that $V$ is 2-dimensional and $\varphi$ acts non-semisimply on $\mathbb{D}_{\text {cris }}(V)$. Thus $\mathbb{D}_{\text {cris }}(V)$ has a basis $\left(e_{1}, e_{2}\right)$ such that $\varphi\left(e_{1}\right)=\alpha e_{1}$ and $\varphi\left(e_{2}\right)=e_{1}+\alpha\left(e_{2}\right)$, for some $\alpha \in E^{\times}$. By twisting, we may assume that the jumps in the Hodge filtration are 0 and $s$ with $s \geq 0$. Let $N$ be the valuation of $\alpha$; the Newton and Hodge numbers of $\mathbb{D}_{\text {cris }}(V)$ are $t_{H}=s$ and $t_{N}=2 N$, so we have $s=2 N$ by weak admissibility.

The unique possible refinement is given by $Y_{1}=E e_{1}$, and this is non-critical unless $s>0$ and Fil ${ }^{1} \mathbb{D}_{\text {cris }}(V)=Y_{1}$. If this is the case, then the Newton and Hodge 
numbers of $Y_{1}$ are respectively $t_{H}\left(Y_{1}\right)=s$ and $t_{N}\left(Y_{1}\right)=N$, and since $s=2 N>N$ this contradicts the weak admissibility of $\mathbb{D}_{\text {cris }}(V)$.

Remark 1.9. (1) It is shown in [Milne 1994] that the Tate conjecture implies the semisimplicity of $\varphi$ on the crystalline cohomology groups of any smooth projective variety over $\mathbb{F}_{p}$ (or, more generally, on the crystalline realisation of any motive over $\mathbb{F}_{p}$ ); so the hypotheses of the proposition conjecturally hold for all crystalline representations "arising from geometry".

(2) For representations of dimension $\geq 3$ with non-semisimple Frobenius there may be no non-critical refinements, as the following counterexample shows. Let $D=\mathbb{Q}_{p}^{3}$ with its standard basis $e_{1}, e_{2}, e_{3}$, and let $\varphi: D \rightarrow D$ be given by the matrix

$$
\left(\begin{array}{ccc}
\alpha & 1 & 0 \\
0 & \alpha & 1 \\
0 & 0 & \alpha
\end{array}\right),
$$

where $\alpha \in \mathbb{Z}_{p}$ has valuation 1 . We define a filtration on $D$ with jumps $\{0,1,2\}$ by $\operatorname{Fil}^{0}(D)=D, \mathrm{Fil}^{1} D=\mathbb{Q}_{p} e_{1}+\mathbb{Q}_{p} e_{3}, \mathrm{Fil}^{2} D=\mathbb{Q}_{p} e_{3}, \mathrm{Fil}^{3} D=0$. Then the only $\varphi$-stable submodules are $Y_{0}=0, Y_{1}=\mathbb{Q}_{p} e_{1}$, and $Y_{2}=\mathbb{Q}_{p} e_{1}+\mathbb{Q}_{p} e_{2}$ and $Y_{3}=D$. The Hodge and Newton numbers are given by

\begin{tabular}{c|c|c}
$i$ & $t_{H}\left(Y_{i}\right)$ & $t_{N}\left(Y_{i}\right)$ \\
\hline 1 & 1 & 1 \\
2 & 1 & 2 \\
3 & 3 & 3
\end{tabular}

so $D$ is a weakly admissible filtered $\varphi$-module; and the unique refinement of $D$ is $\left(Y_{i}\right)_{i=0, \ldots, 3}$, but $Y_{1}$ is not in general position.

1C6. Modular forms. Let $f(z)=\sum a_{n} e^{2 \pi i n z}$ be a normalised new eigenform of weight $k \geq 2$, level $N$ and nebentypus $\varepsilon$. Write $F_{f}=\mathbb{Q}\left(a_{n}: n \geq 1\right)$ for its coefficient field. Let

$$
\bar{f}(z)=\sum \bar{a}_{n} e^{2 \pi i n z}
$$

be the dual form to $f$, which also has coefficients in $F_{f}$. We assume that $p \nmid N$ and $a_{p}$ is not a $p$-adic unit, so $f$ is supersingular at $p$.

Remark 1.10. We make this assumption in order to save ourselves from doing the same calculations twice in Section 5; they easily generalise to the ordinary case.

We fix a prime of $F_{f}$ above $p$. We denote the completion of $F_{f}$ at this prime by $E$ and fix a uniformiser $\varpi_{E}$. We write $V_{f}$ for the 2-dimensional $E$-linear representation of $G_{\mathbb{Q}}$ associated to $f$ from [Deligne 1971]. We fix an $\mathscr{O}_{E}$-lattice $T_{f}$ 
stable under $G_{\mathbb{Q}_{\mathbb{Q}}}$, which determines a lattice $T_{\bar{f}}$ of $V_{\bar{f}}$. When restricted to $G_{\mathbb{Q}_{p}}, V_{f}$ is crystalline and its de Rham filtration is given by

$$
\operatorname{dim}_{E} \text { Fil }^{i} \mathbb{D}_{\text {cris }}\left(V_{f}\right)= \begin{cases}2 & \text { if } i \leq 0 \\ 1 & \text { if } 1 \leq i \leq k-1, \\ 0 & \text { if } i \geq k\end{cases}
$$

The action of $\varphi$ on $\mathbb{D}_{\text {cris }}\left(V_{f}\right)$ satisfies $\varphi^{2}-a_{p} \varphi+\varepsilon(p) p^{k-1}=0$. Let us choose a "good basis" $v_{1}, v_{2}$ of $\mathbb{D}_{\text {cris }}\left(V_{f}\right)$ as in [Lei et al. 2010, §3.3]; that is, $v_{1}$ spans Fil $1^{1} \mathbb{D}_{\text {cris }}\left(V_{f}\right)$ and $v_{2}=p^{1-k} \varphi\left(v_{1}\right)$. We also choose a basis $\bar{v}_{1}, \bar{v}_{2}$ of $\mathbb{D}_{\text {cris }}\left(V_{\bar{f}}\right)$ in the same way. The isomorphism $V_{\bar{f}}=V_{f}^{*}(1-k)$ gives a pairing $\mathbb{D}_{\text {cris }}\left(V_{f}\right) \times$ $\mathbb{D}_{\text {cris }}\left(V_{\bar{f}}\right) \rightarrow \mathbb{D}_{\text {cris }}(E(1-k))=E \cdot t^{k-1} e_{1-k} \cong E$. As noted in [Lei et al. 2010, $\S 3.4]$, we have $\left[v_{1}, \bar{v}_{1}\right]=\left[v_{2}, \overline{v_{2}}\right]=0$ and $\left[v_{2}, \bar{v}_{1}\right]=-\left[v_{1}, \bar{v}_{2}\right]$, and (by scaling) we may assume without loss of generality that $\left[v_{1}, \bar{v}_{2}\right]=1$.

Unless otherwise stated, we always assume that the eigenvalues of $\varphi$ on $\mathbb{D}_{\text {cris }}\left(V_{f}\right)$ are not integral powers of $p$ and the nebentypus of $f$ is trivial. Our assumption on the eigenvalues of $\varphi$ allows us to define the Perrin-Riou pairing

$$
\mathscr{L}_{i}=\mathscr{L}_{1,(1+\pi) \otimes v_{i, 1}}: H_{\mathrm{IW}}^{1}\left(\mathbb{Q}_{p}, V_{\bar{f}}(k-1)\right) \rightarrow \mathscr{H}(\Gamma)
$$

for $i=1,2$ where we have identified $V_{f}(1)^{*}(1)$ with $V_{\bar{f}}(k-1)$ (see [Lei 2011, Section 3.2] or [Lei et al. 2010, Section 3.3] for details).

1C7. Adequate rings and elementary divisors. Let $R$ be a commutative integral domain with identity, such that the following conditions hold:

- All finitely generated ideals in $R$ are principal (i.e., $R$ is a Bézout domain).

- $R$ is adequate; i.e., for any $a, b \in R$ with $a \neq 0$, we may write $a=a_{1} a_{2}$, where $\left(a_{1}, b\right)=(1)$ and $(d, b) \neq(1)$ for every non-unit divisor $d$ of $a_{2}$.

Then $R$ is an elementary divisor ring. That is, let $M \subseteq N$ be finitely generated $R$-modules such that $N \cong R^{d}$. Then there exists a $R$-basis $n_{1}, \ldots, n_{d}$ of $N$ and $r_{1}, \ldots, r_{d} \in R$ (unique up to units of $R$ ) such that $r_{1}|\cdots| r_{d}$ and $r_{1} n_{1}, \ldots, r_{e} n_{e}$, where $e$ is the largest integer such that $r_{e} \neq 0$, form a $R$-basis of $M$. In particular, we have $\operatorname{det}(M)=r_{1} \ldots r_{d}$. In this case, we write $[N: M]=[N: M]_{R}=\left[r_{1} ; \ldots ; r_{d}\right]$. When $d=1$, we simply write $[N: M]=r_{1}$.

If $Q$ is an arbitrary finitely presented $R$-module, then we may write $Q$ as a quotient $N / M$ where $N$ is a free module of finite rank and $M$ is a finitely generated submodule of $N$, so the elementary divisors $[N: M]_{R}$ are defined. It is easy to check that these are independent of the choice of presentation of $Q$, and we define these to be the elementary divisors of $Q$.

As explained in [Berger 2002, §4.2], $\mathbb{B}_{\text {rig, } \mathbb{Q}_{p}}^{+}$is an adequate Bézout domain and hence an elementary divisor ring. The same is true of $E \otimes_{\mathbb{Q}_{p}} \mathbb{B}_{\text {rig, } \mathbb{Q}_{p}}^{+}$for any finite 
extension $E$ of $\mathbb{Q}_{p}$, and of $\mathscr{H}\left(\Gamma_{1}\right)$ (which is isomorphic to $\mathbb{B}_{\text {rig, } \mathbb{Q}_{p}}^{+}$as an abstract ring).

We will need the following lemma; see [Lang 2002, Lemma III.7.6].

Lemma 1.11. Let $R$ be an adequate Bézout domain, $M$ a finitely presented $R$ module, and $N$ a submodule of $M$. Suppose that there is some $a \in R$ such that $N \cong R / a$ and $a M=0$. Then $M \cong N \oplus M / N$.

Proof. Let $q_{1}, \ldots, q_{r}$ be a set of generators for $M / N$, with annihilators $a_{i}$, giving an isomorphism $M / N \cong \oplus_{i=1}^{r} R / a_{i}$. Since $a M=0$, each $a_{i}$ divides $a$. Let $p_{i}$ be an arbitrary lift of $q_{i}$; then $a_{i} p_{i} \in N$, so $a_{i} p_{i}=b_{i} p_{0}$ where $p_{0}$ is a generator of $N$ and $b_{i} \in R / a R$. Since $a M=0$, we have $0=\left(a / a_{i}\right) a_{i} p_{i}=\left(a / a_{i}\right) b_{i} p_{0}$.

Then we must have $\left(a / a_{i}\right) b_{i} \in a R$, so $a b_{i} \in a a_{i} R$. Since $R$ is an integral domain, we must have $a_{i} \mid b_{i}$, and we may write $b_{i}=a_{i} c_{i}$. Thus $p_{i}^{\prime}=p_{i}-c_{i} p_{0}$ is a lift of $p_{i}$ such that $a_{i} p_{i}^{\prime}=a_{i} p_{i}-a_{i} c_{i} p_{0}=a_{i} p_{i}-b_{i} p_{0}=0$. It follows that the subgroup generated by the $p_{i}^{\prime}$ maps bijectively to $M / N$, giving the required splitting.

A straightforward induction gives the following generalisation:

Corollary 1.12. If $M$ is an R-module with a filtration by submodules $0=M_{0} \subseteq$ $M_{1} \subseteq \cdots \subseteq M_{d}=M$, and there are elements $a_{1}, \ldots, a_{d} \in R$ such that for each $i=1, \ldots, d$ we have $M_{i} / M_{i-1} \cong R / a_{i}$ and $a_{i} M \subseteq M_{i-1}$, then $M \cong \bigoplus_{i=1}^{d} R / a_{i}$.

The ring $\mathscr{H}(\Gamma)$ is not a domain; but it is equal to the direct sum of its subrings $e_{\eta} \mathscr{H}(\Gamma)$, where $e_{\eta}$ is the idempotent in $\mathbb{Q}_{p}[\Delta]$ corresponding to the character $\eta$ : $\Delta \rightarrow \mathbb{Q}_{p}^{\times}$as above. Each of these subrings is isomorphic to $\mathscr{H}\left(\Gamma_{1}\right)$, and hence admits a theory of elementary divisors. If $M$ is a submodule of $\mathscr{H}(\Gamma)^{\oplus d}$, we define the $i$ th elementary divisor of $M$ to be $\sum_{\eta} e_{\eta} a_{i}^{\eta}$, where $a_{i}^{\eta}$ is the $i$ th elementary divisor of the submodule $M^{\eta}=e_{\eta} M \subseteq e_{\eta} \mathscr{H}(\Gamma)$ considered as a $\mathscr{H}\left(\Gamma_{1}\right)$-module. In practice we shall only apply this in situations where $M$ has the form $\mathbb{Q}_{p}[\Gamma] \otimes_{\mathbb{Q}_{p}} M^{\prime}$ for an $\mathscr{H}\left(\Gamma_{1}\right)$-module $M$, in which case the isotypical components $M^{\eta}$ all have the same elementary divisors.

\section{Refinements of crystalline representations and $\mathscr{H}(\Gamma)$-structure}

In this section, we will prove Theorem A. We will do this by working with a certain filtration of the module $\mathbb{N}_{\text {rig }}(V)$, which is a $(\varphi, \Gamma)$-module over $\mathbb{B}_{\text {rig, } \mathbb{Q}_{p}}^{+}$; the steps in this filtration are $(\varphi, \Gamma)$-modules over $\mathbb{B}_{\text {rig, } \mathbb{Q}_{p}}^{+}$, but they are not necessarily of the form $\mathbb{N}_{\text {rig }}(W)$ for any representation $W$, so we begin by systematically developing a theory of such modules. Our approach is very much influenced by the description of the theory of $(\varphi, \Gamma)$-modules over the Robba ring $\mathbb{B}_{\text {rig, } \mathbb{Q}_{p}}^{\dagger}$ given in [Bellaïche and Chenevier 2009, §2.2]. 
2A. Some properties of $(\varphi, \Gamma)$-modules over $\mathbb{B}_{\text {rig, } \mathbb{Q}_{p}}^{+}$. We define a $(\varphi, \Gamma)$-module over $\mathbb{B}_{\text {rig, } \mathbb{Q}_{p}}^{+}$to be a free $\mathbb{B}_{\text {rig, } \mathbb{Q}_{p}}^{+}$-module $\mathcal{N}$ of finite rank, endowed with semilinear commuting actions of $\varphi$ and $\Gamma$, such that the quotient $\mathcal{N} / \varphi^{*}(\mathcal{N})$ is annihilated by some power of $q$ (where $q=\varphi(\pi) / \pi$ as above). We define

$$
\mathbb{D}_{\text {cris }}(\mathcal{N})=\mathcal{N}^{\Gamma} \text {. }
$$

We equip $\mathbb{D}_{\text {cris }}(\mathcal{N})$ with the filtration defined by

$$
\text { Fil }^{i} \mathbb{D}_{\text {cris }}(\mathcal{N})=\left\{v \in \mathbb{D}_{\text {cris }}(\mathcal{N}): \varphi(v) \in q^{i} \mathcal{N}\right\}
$$

Let $K_{n}=\mathbb{Q}_{p}\left(\mu_{p^{n}}\right)$ and $K_{\infty}=\bigcup_{n} K_{n}$. We define

$$
\mathbb{D}_{\mathrm{dR}}^{(n)}(\mathcal{N})=\left(K_{\infty} \otimes_{K_{n}} K_{n} \llbracket t \rrbracket \otimes_{\mathbb{B}_{\mathrm{rig}, \mathbb{Q}_{p}}^{+}} \mathcal{N}\right)^{\Gamma},
$$

where the tensor product is via the embedding $\mathbb{B}_{\text {rig, } \mathbb{Q}_{p}}^{+} \hookrightarrow K_{n} \llbracket t \rrbracket$ arising from the fact that

$$
K_{n} \cong \mathbb{B}_{\text {rig, } \mathbb{Q}_{p}}^{+} / \varphi^{n-1}(q)
$$

and $t$ is a uniformiser of the prime ideal $\varphi^{n-1}(q)$. We endow $K_{n} \llbracket t \rrbracket$ with the obvious semilinear action of $\Gamma$, for which this homomorphism is $\Gamma$-equivariant, and the $t$-adic filtration. Then $\mathbb{D}_{\mathrm{dR}}^{(n)}(\mathcal{N})$ is a filtered $\mathbb{Q}_{p}$-vector space, of dimension $\leq d$ where $d$ is the $\mathbb{B}_{\text {rig, } \mathbb{Q}_{p}}^{+}$-rank of $\mathcal{N}$ (since $K_{\infty}((t))^{\Gamma}=\mathbb{Q}_{p}$ [Bellaïche and Chenevier $2009, \S 2.2 .7])$; the operator $\varphi$ gives an isomorphism of filtered $\mathbb{Q}_{p}$-vector spaces

$$
\mathbb{D}_{\mathrm{dR}}^{(n)}(\mathcal{N}) \stackrel{\cong}{\longrightarrow} \mathbb{D}_{\mathrm{dR}}^{(n+1)}(\mathcal{N})
$$

for each $n$, and an embedding of filtered $\mathbb{Q}_{p}$-vector spaces

$$
\mathbb{D}_{\text {cris }}(\mathcal{N}) \hookrightarrow \mathbb{D}_{\mathrm{dR}}^{(1)}(\mathcal{N}) .
$$

We say that $\mathcal{N}$ is crystalline if $\operatorname{dim}_{\mathbb{Q}_{p}} \mathbb{D}_{\text {cris }}(\mathcal{N})=d$, and we say it is de Rham if $\operatorname{dim}_{\mathbb{Q}_{p}} \mathbb{D}_{\mathrm{dR}}^{(n)}(\mathcal{N})=d$ (for some, and hence all, $n \geq 1$ ). If $\mathcal{N}$ is de Rham, we define the Hodge-Tate weights of $\mathcal{N}$ to be the integers $r$ such that $\mathrm{Fil}^{-r} \mathbb{D}_{\mathrm{dR}}^{(n)}(\mathcal{N}) \neq$ Fil $^{1-r} \mathbb{D}_{\mathrm{dR}}^{(n)}(\mathcal{N})$ (with multiplicities given by the size of the jump in dimension). Note that these are necessarily $\leq 0$, which is unfortunate but necessary for compatibility with the usual definition in the case of Galois representations.

Finally, we define $\mathbb{D}_{\mathrm{Sen}}^{(n)}(\mathcal{N})=K_{\infty} \otimes_{K_{n}} \mathcal{N} / \varphi^{n-1}(q) \mathcal{N}$. This is a $K_{\infty}$-vector space of dimension $d$, with a semilinear action of $\Gamma$. As above, the $\varphi$ operator gives isomorphisms $\mathbb{D}_{\mathrm{Sen}}^{(n)}(\mathcal{N}) \rightarrow \mathbb{D}_{\mathrm{Sen}}^{(n+1)}(\mathcal{N})$, of $K_{\infty}$-vector spaces with semilinear $\Gamma$ action. (So both $\mathbb{D}_{\text {Sen }}(\mathcal{N})$ and $\mathbb{D}_{\mathrm{dR}}(\mathcal{N})$ are independent of $n$ as abstract objects; we retain the $n$ in the notation when we are interested in the relation between these spaces and the original module $\mathcal{N}$.) 
Proposition 2.1. Let $j \geq 0$, and suppose $\mathcal{N}$ is de Rham. Then there is an isomorphism of $\mathbb{Q}_{p}$-vector spaces

$$
\operatorname{Fil}^{j} \mathbb{D}_{\mathrm{dR}}(\mathcal{N}) / \mathrm{Fil}^{j+1} \mathbb{D}_{\mathrm{dR}}(\mathcal{N}) \stackrel{\cong}{\longrightarrow} \mathbb{D}_{\mathrm{Sen}}(\mathcal{N})^{\Gamma=\chi^{-j}} .
$$

Proof. Let us fix an $n \geq 1$ and let $\theta$ be the reduction map $K_{n} \llbracket t \rrbracket \rightarrow K_{n}$. Then $\theta$ induces a map

$$
\mathbb{D}_{\mathrm{dR}}^{(n)}(\mathcal{N}) \rightarrow \mathbb{D}_{\mathrm{Sen}}^{(n)}(\mathcal{N})^{\Gamma}
$$

with kernel Fil ${ }^{1} \mathbb{D}_{\mathrm{dR}}(\mathcal{N})$ and whose image is a $\mathbb{Q}_{p}$-linear subspace $S_{0} \subseteq \mathbb{D}_{\operatorname{Sen}}(\mathcal{N})^{\Gamma}$. Similarly, we find that $\theta \circ t^{-j}$ gives an injection

$$
\operatorname{Fil}^{j} \mathbb{D}_{\mathrm{dR}}(\mathcal{N}) / \mathrm{Fil}^{j+1} \mathbb{D}_{\mathrm{dR}}(\mathcal{N}) \rightarrow \mathbb{D}_{\mathrm{Sen}}(\mathcal{N})^{\Gamma=\chi^{-j}},
$$

whose image is a $\mathbb{Q}_{p}$-linear subspace $S_{j}$.

Since $\bigoplus_{j=0}^{\infty} S_{j}$ has dimension $d$, it suffices to show that

$$
\operatorname{dim}_{\mathbb{Q}_{p}} \bigoplus_{j=0}^{\infty} \mathbb{D}_{\operatorname{Sen}}(\mathcal{N})^{\Gamma=\chi^{-j}} \leq d .
$$

This follows from the fact that it is a subspace of $\left(K_{\infty}((t)) \otimes_{K_{\infty}} \mathbb{D}_{\text {Sen }}(\mathcal{N})\right)^{\Gamma}$, and (as remarked above) $\left.K_{\infty}((t))\right)$ is a field, with $K_{\infty}((t))^{\Gamma}=\mathbb{Q}_{p}$.

Corollary 2.2. If $\mathcal{N}$ is crystalline, then the map

$$
\mathbb{D}_{\text {cris }}(\mathcal{N})=\mathcal{N}^{\Gamma} \stackrel{\varphi^{n}}{\longrightarrow}\left(\mathcal{N} / \varphi^{n-1}(q)^{r} \mathcal{N}\right)^{\Gamma}
$$

is surjective for all $r \geq 1$ and $n \geq 1$, with kernel Fil $^{r} \mathbb{D}_{\text {cris }}(\mathcal{N})$.

Proof. Let us define $\mathcal{N}^{(n)}=K_{\infty} \otimes_{K_{n}} K_{n} \llbracket t \rrbracket \otimes_{\mathbb{B}_{\text {rig, } \mathbb{Q}_{p}}^{+}} \mathcal{N}$, so $\left(\mathcal{N}^{(n)}\right)^{\Gamma}=\mathbb{D}_{\mathrm{dR}}(\mathcal{N})$. By hypothesis the map $\varphi^{n}: \mathbb{D}_{\text {cris }}(\mathcal{N}) \rightarrow \mathbb{D}_{\mathrm{dR}}^{(n)}(\mathcal{N})$ is an isomorphism of filtered vector spaces, and the filtration on $\mathbb{D}_{\mathrm{dR}}(\mathcal{N})$ is defined by the $t$-adic filtration of $\mathcal{N}^{(n)}$, so it suffices to show that reduction modulo $t^{r}$ gives a surjection

$$
\left(\mathcal{N}^{(n)}\right)^{\Gamma} \rightarrow\left(\mathcal{N}^{(n)} / t^{r} \mathcal{N}^{(n)}\right)^{\Gamma} .
$$

We show that for each $j$, the map $\left(t^{j} \mathcal{N}^{(n)}\right)^{\Gamma} \rightarrow\left(t^{j} \mathcal{N}^{(n)} / t^{j+1} \mathcal{N}^{(n)}\right)^{\Gamma}$ is surjective. Multiplication by $t^{-j}$ gives an isomorphism

$$
\left(t^{j} \mathcal{N}^{(n)} / t^{j+1} \mathcal{N}^{(n)}\right)^{\Gamma} \rightarrow\left(\mathcal{N}^{(n)} / t \mathcal{N}^{(n)}\right)^{\Gamma=\chi^{-j}}
$$

but $\mathcal{N}^{(n)} / t \mathcal{N}^{(n)}=\mathbb{D}_{\mathrm{Sen}}^{(n)}(\mathcal{N})$, and by the preceding proposition we know that $\theta \circ t^{-j}$ gives an isomorphism from $\mathrm{Fil}^{j} \mathbb{D}_{\mathrm{dR}}(\mathcal{N}) / \mathrm{Fil}^{j+1} \mathbb{D}_{\mathrm{dR}}(\mathcal{N})$ to $\mathbb{D}_{\mathrm{Sen}}^{(n)}(\mathcal{N})^{\Gamma=\chi^{-j}}$. So the map $\left(\mathcal{N}^{(n)}\right)^{\Gamma} \rightarrow\left(\mathcal{N}^{(n)} / t^{r} \mathcal{N}^{(n)}\right)^{\Gamma}$ is a morphism of filtered vector spaces for which the associated map of graded modules is surjective. Since the domain and codomain 
are finite-dimensional and their filtrations are separated, the original map is itself surjective.

Let us write $\mathcal{M}=\mathbb{B}_{\text {rig, } \mathbb{Q}_{p}}^{+} \otimes_{\mathbb{Q}_{p}} \mathbb{D}_{\text {cris }}(\mathcal{N}) \subseteq \mathcal{N}$.

Proposition 2.3. If $\mathcal{N}$ is crystalline and $\Gamma$ acts trivially on $\mathcal{N} / \pi \mathcal{N}$, then the elementary divisors of $\mathcal{N} / \mathcal{M}$ are

$$
\left(\frac{t}{\pi}\right)^{s_{1}}, \ldots,\left(\frac{t}{\pi}\right)^{s_{d}},
$$

where $-s_{1} \geq \cdots \geq-s_{d}$ are the Hodge-Tate weights of $\mathcal{N}$.

Proof. This follows exactly as in [Berger 2004, Proposition III.2.1].

2B. Quotients of $(\varphi, \Gamma)$-modules. We now let $\mathcal{N}$ be a $(\varphi, \Gamma)$-module over $\mathbb{B}_{\text {rig, } \mathbb{Q}_{p}}^{+}$, as above. We assume that $\mathcal{N}$ is crystalline and $\Gamma$ acts trivially on $\mathcal{N} / \pi \mathcal{N}$, and investigate the properties of a certain class of $(\varphi, \Gamma)$-modules obtained as quotients of $\mathcal{N}$. We continue to write $M=\mathbb{B}_{\text {rig, } \mathbb{Q}_{p}}^{+} \otimes_{\mathbb{Q}_{p}} \mathbb{D}_{\text {cris }}(\mathcal{N}) \subseteq \mathcal{N}$.

Let $Y$ be a $\varphi$-stable $E$-linear subspace of $\mathbb{D}_{\text {cris }}(\mathcal{N})$. We set

$$
\mathscr{Y}=\mathbb{B}_{\text {rig, }, \mathbb{Q}_{p}}^{+} \otimes_{\mathbb{Q}_{p}} Y \subseteq \mathcal{M} \text {. }
$$

and

$$
\mathscr{X}=\mathcal{N} \cap \mathcal{Y}\left[\left(\frac{t}{\pi}\right)^{-1}\right]=\left\{x \in \mathcal{N}:\left(\frac{t}{\pi}\right)^{m} x \in \mathscr{Y} \text { for some } m\right\} \subseteq \mathcal{N} .
$$

Proposition 2.4. The spaces $Y, \mathscr{Y}, \mathscr{X}$ have the following properties:

(a) $\mathscr{X}$ is a $\mathbb{B}_{\mathrm{rig}, \mathbb{Q}_{p}}^{+}$-submodule of $\mathcal{N}$ stable under $\varphi$ and $\Gamma$;

(b) $\mathscr{X}=\left\{x \in \mathcal{N}: a x \in \mathscr{Y}\right.$ for some nonzero $\left.a \in \mathbb{B}_{\text {rig, } \mathbb{Q}_{p}}^{+}\right\}$(the saturation of $\mathscr{Y}$ );

(c) $\mathscr{X}$ is free of rank $\operatorname{dim}_{\mathbb{Q}_{p}} Y$ as an $\mathbb{B}_{\text {rig, } \mathbb{Q}_{p}}^{+}$-module;

(d) $Y=\mathscr{X} \cap \mathbb{D}_{\text {cris }}(\mathcal{N})$ and $\mathscr{Y}=\mathscr{X} \cap M$;

(e) $\mathscr{X}$ and $\mathscr{W}=\mathcal{N} / \mathscr{X}$ are $(\varphi, \Gamma)$-modules over $\mathbb{B}_{\text {rig, } \mathbb{Q}_{p}}^{+}$.

Proof. Part (a) is immediate from the definition.

For (b), suppose $x \in \mathcal{N}$ and there is some nonzero $a \in \mathbb{B}_{\text {rig, } \mathbb{Q}_{p}}^{+}$such that $a x \in \mathcal{Y}$. By Proposition 2.3, we can find $m$ such that $\left(\frac{t}{\pi}\right)^{m} x \in \mathcal{M}$, and $a\left(\frac{t}{\pi}\right)^{m} x \in \mathcal{Y}$. Since $y$ is clearly saturated in $\mathcal{M}$, we deduce that $\left(\frac{t}{\pi}\right)^{m} x \in \mathscr{Y}$, and hence $x \in \mathscr{X}$ as required.

For part (c), we note that $\mathscr{X}$ is a closed submodule of $\mathcal{N}$, since it is the intersection of the closed submodules $\left(\frac{t}{\pi}\right)^{-N}$ y and $\mathcal{N}$ of $\left(\frac{t}{\pi}\right)^{-N} \mathcal{N}$, for any sufficiently large $N$. (It suffices to take $N$ larger than $s_{d}$, where $-s_{d}$ is the lowest Hodge-Tate weight of $\mathcal{N}$.) Hence $\mathscr{X}$ is also a free module, of finite rank. As $\mathscr{X}\left[\left(\frac{t}{\pi}\right)^{-1}\right]$ is clearly free

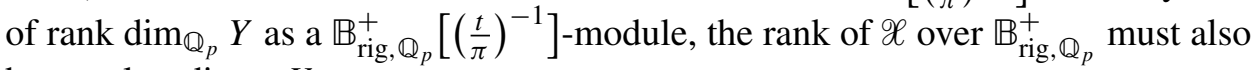
be equal to $\operatorname{dim}_{\mathbb{Q}_{p}} Y$.

For part (d), it is clear that $\mathscr{Y} \subseteq \mathscr{X} \cap \mathcal{M}$; and this inclusion is an equality, since $\mathcal{M} / \mathscr{Y}$ is torsion-free and $\mathscr{X} / \mathscr{Y}$ is torsion. Since $\mathscr{Y} \cap \mathbb{D}_{\text {cris }}(\mathcal{N})=Y$, the statement follows. 
For the final statement (e), since $\mathscr{X}$ and $\mathscr{W}=\mathcal{N} / \mathscr{X}$ are both free $\mathbb{B}_{\text {rig, } \mathbb{Q}_{p}}^{+}-$modules with semilinear actions of $\varphi$ and $\Gamma$, it suffices to check that the modules $\mathscr{X} / \varphi^{*} \mathscr{X}$ and $\mathscr{W} / \varphi^{*} \mathscr{W}$ are annihilated by a power of $q$. Since $\mathscr{X}$ is saturated in $\mathcal{N}$, and $\mathbb{B}_{\text {rig, } \mathbb{Q}_{p}}^{+}$ is an elementary divisor ring, we can find a $\mathbb{B}_{\text {rig, } \mathbb{Q}_{p}}^{+}$-basis $n_{1}, \ldots, n_{d}$ of $\mathcal{N}$ such that $n_{1}, \ldots, n_{r}$ is a basis of $\mathscr{X}$ and the images of $n_{r+1}, \ldots, n_{d}$ are a basis of $\mathscr{W}$, where $r=\operatorname{dim}_{\mathbb{Q}_{p}} Y$. Since $\mathscr{X}$ is $\varphi$-stable, the matrix of $\varphi$ in this basis is of the form $\left(\begin{array}{cc}A & B \\ 0 & C\end{array}\right)$. Hence we have $\operatorname{det}\left(\varphi^{*} \mathcal{N}\right)=\operatorname{det}(A) \operatorname{det}(C)$. As $\mathcal{N} / \varphi^{*} \mathcal{N}$ is annihilated by a power of $q, \operatorname{det}\left(\varphi^{*} \mathcal{N}\right)$ is a power of $q$, and thus the same is true of $\operatorname{det}(A)$ and $\operatorname{det}(C)$. Since $A$ and $C$ are the matrices of $\varphi$ on $\mathscr{X}$ and $\mathscr{W}$ in the bases described above, the modules $\mathscr{X} / \varphi^{*} \mathscr{X}$ and $\mathscr{W} / \varphi^{*} \mathscr{W}$ are also annihilated by a power of $q$, as required.

Let $W=\mathbb{D}_{\text {cris }}(\mathcal{N}) / Y$, and (as above) let $\mathscr{W}=\mathcal{N} / \mathscr{X}$. The natural map $W \hookrightarrow$ $\mathbb{D}_{\text {cris }}(\mathcal{W})$ is injective, by part $(\mathrm{d})$ of the preceding proposition; hence it is also surjective, for reasons of dimension. Thus $\mathscr{W}$ is a crystalline $(\varphi, \Gamma)$-module and $\mathbb{D}_{\text {cris }}(\mathscr{W})=W$.

Proposition 2.5. The quotient filtration Fil ${ }^{\bullet} W$ induced on $W$ by the filtration of $\mathbb{D}_{\text {cris }}(N)$ agrees with the filtation Fil given by

$$
\underline{\mathrm{Fil}}^{r} W=\left\{w \in W: \varphi(w) \in q^{r} W\right\} .
$$

Proof. It is clear from the definition that $\mathrm{Fil}^{r} W \subseteq \underline{\mathrm{Fil}}^{r} W$.

Conversely, let $y \in \mathbb{D}_{\text {cris }}(N)$ such that $[y] \in \underline{\text { Fil }}^{r} W$, so we can write $\varphi(y)=$ $q^{r} y^{\prime}+z$ for some $y^{\prime} \in N$ and $z \in \mathscr{X}$. Then

$$
z \bmod q^{r} \mathscr{X} \in\left(\mathscr{X} / q^{r} \mathscr{X}\right)^{\Gamma} .
$$

Applying Corollary 2.2 to $\mathscr{X}$, we find that $z$ is congruent modulo $q^{r}$ to an element of $\mathscr{L}^{\Gamma}=Y$.

The final result we will need about these quotients is the following slightly fiddly lemma. Let us suppose that the jumps in the filtration of $\mathbb{D}_{\text {cris }}(\mathcal{N})$, with multiplicity, are $s_{1} \leq s_{2} \cdots \leq s_{d}$ (i.e., the Hodge-Tate weights of $\mathcal{N}$ are $-s_{i}$ ). We say that the $\varphi$-stable subspace $Y$ is in general position (with respect to the Hodge filtration of $\left.\mathbb{D}_{\text {cris }}(\mathcal{N})\right)$ if the jumps in the filtration Fil $l^{\bullet}$ are $s_{1}, \ldots, s_{j}$, where $j=\operatorname{dim}_{\mathbb{Q}_{p}} Y$.

Lemma 2.6. If $Y$ is in general position, then for any $m \geq s_{d}$, we have

$$
\left(\frac{t}{\pi}\right)^{m-s_{(j+1)}} \mathcal{M} \subseteq\left(\frac{t}{\pi}\right)^{m} \mathcal{N}+\mathscr{Y} .
$$

Proof. As remarked above, the quotient module $\mathscr{W}=\mathcal{N} / \mathscr{X}$ is a crystalline $(\varphi, \Gamma)$ module over $\mathbb{B}_{\text {rig, } \mathbb{Q}_{p}}^{+}$of rank $d-j$, with $\Gamma$ acting trivially modulo $\pi$. By Proposition 2.5 , the Hodge-Tate weights of $\mathcal{W}$ are exactly $\left\{-s_{(j+1)}, \ldots,-s_{d}\right\}$; hence its $\Gamma$ invariants lie in $\left(\frac{t}{\pi}\right)^{s_{(j+1)}} \mathscr{W}$. This is equivalent to $\mathcal{M} \subseteq\left(\frac{t}{\pi}\right)^{s_{(j+1)}} \mathcal{N}+\mathscr{X}$. Multiplying 
by $\left(\frac{t}{\pi}\right)^{m-s_{(j+1)}}$, we see that

$$
\left(\frac{t}{\pi}\right)^{m-s_{(j+1)}} \mathcal{M} \subseteq\left(\frac{t}{\pi}\right)^{m} \mathcal{N}+\left(\frac{t}{\pi}\right)^{m-s_{(j+1)}} \mathscr{X} .
$$

Since both $\left(\frac{t}{\pi}\right)^{m-s_{(j+1)}} \mathcal{M}$ and $\left(\frac{t}{\pi}\right)^{m} \mathcal{N}$ are manifestly contained in $\mathcal{M}$, we may replace the last term with its intersection with $\mathcal{M}$, which is clearly contained in $\mathscr{X} \cap M=\mathscr{Y}$.

2C. Application to crystalline representations. Let $V$ be a $d$-dimensional crystalline representation of $G_{\mathbb{Q}_{p}}$ with Hodge-Tate weights $\left\{-s_{1}, \ldots,-s_{d}\right\}$, where $0 \leq s_{1} \leq \cdots \leq s_{d}$ (so $V$ is positive in the sense of Section 1C4 above). As above, we define $\mathbb{N}_{\text {rig }}(V)=\mathbb{B}_{\text {rig, } \mathbb{Q}_{p}}^{+} \otimes_{\mathbb{B}_{\mathbb{Q}_{p}}^{+}} \mathbb{N}(V)$, where $\mathbb{N}(V)$ is the Wach module of $V$ as constructed in [Berger 2004]. Then $\mathbb{N}_{\text {rig }}(V)$ is a crystalline $(\varphi, \Gamma)$-module over $\mathbb{B}_{\text {rig, } \mathbb{Q}_{p}}^{+}$with $\Gamma$ acting trivially modulo $\pi$, and $\mathbb{D}_{\text {cris }}(V)$ is isomorphic (as a filtered $\varphi$-module over $\left.\mathbb{Q}_{p}\right)$ to $\mathbb{D}_{\text {cris }}\left(\mathbb{N}_{\text {rig }}(V)\right)$ as defined in the previous section [Berger 2004, Theorems II.2.2 and III.4.4].

If $V$ is in fact an $E$-linear representation, for $E$ some finite extension of $\mathbb{Q}_{p}$, then $\mathbb{N}_{\text {rig }}(V)$ is naturally an $E \otimes_{\mathbb{Q}_{p}} \mathbb{B}_{\text {rig, } \mathbb{Q}_{p}}^{+}$-module, and $\mathbb{D}_{\text {cris }}(V)$ is a filtered $E$ vector space. If we choose an $E$-linear $\varphi$-stable subspace, then all of the above constructions commute with the additional $E$-linear structure.

We shall suppose that $V$ admits a non-critical refinement, and fix a choice of such a refinement $\underline{Y}$. Applying the above theory to each of the subspaces $Y_{i}$, we obtain $E \otimes_{\mathbb{Q}_{p}} \mathbb{B}_{\text {rig, } \mathbb{Q}_{p}}^{+}$-submodules $\mathscr{Y}_{i}=\mathbb{B}_{\text {rig, } \mathbb{Q}_{p}}^{+} \otimes_{\mathbb{Q}_{p}} Y_{i} \subseteq \mathcal{M}$ and $\mathscr{L}_{i}=\mathscr{Y}_{i}^{\text {sat }}$ of $\mathbb{N}_{\text {rig }}(V)$.

Let us consider the representation $V(m)$, for some $m \geq s_{d}$. This has non-negative Hodge-Tate weights $\left\{m-s_{i}\right\}_{i=1, \ldots, d}$. If $e_{m}$ denotes a basis of $\mathbb{Q}_{p}(m)$, then we have

$$
\begin{aligned}
\mathbb{D}_{\text {cris }}(V(m)) & =\left\{t^{-m} x \otimes e_{m}: x \in \mathbb{D}_{\text {cris }}(V)\right\}, \\
\mathbb{N}_{\text {rig }}(V(m)) & =\left\{\pi^{-m} y \otimes e_{m}: y \in \mathbb{N}_{\text {rig }}(V)\right\} .
\end{aligned}
$$

We define $\mathscr{A}_{i}=\left\{\pi^{-m} y \otimes e_{m}: y \in \mathscr{X}_{i}\right\}$ and $\mathscr{B}_{i}=\left\{t^{-m} x \otimes e_{m}: x \in \mathscr{Y}_{i}\right\}$.

Proposition 2.7. For each $i=1, \ldots, d$,

(a) $\left(\frac{t}{\pi}\right)^{m-s_{i}} \mathscr{B}_{i} \supseteq \mathscr{A}_{i} \supseteq\left(\frac{t}{\pi}\right)^{m-s_{1}} \mathscr{B}_{i}$;

(b) $\mathscr{B}_{i}$ is the saturation of $\mathscr{A}_{i}$ in $\mathscr{B}_{d}=\mathbb{B}_{\text {rig, } \mathbb{Q}_{p}}^{+} \otimes \mathbb{D}_{\text {cris }}(V(m))$;

(c) The inclusion $\mathscr{A}_{d} \hookrightarrow \mathscr{B}_{d}$ identifies $\mathscr{A}_{d} / \mathscr{A}_{i-1}$ with a submodule of $\mathscr{B}_{d} / \mathscr{B}_{i-1}$ and the quotient is annihilated by $\left(\frac{t}{\pi}\right)^{m-s_{i}}$.

Proof. The chain of inclusions in (a) is equivalent to $\left(\frac{t}{\pi}\right)^{s_{1}} \mathscr{X}_{i} \supseteq \mathscr{Y}_{i} \supseteq\left(\frac{t}{\pi}\right)^{s_{i}} \mathscr{X}_{i}$, and this is a consequence of Proposition 2.3 since the Hodge-Tate weights of $\mathscr{X}_{i}$ are $\left\{-s_{1}, \ldots,-s_{i}\right\}$. Moreover, $\mathscr{B}_{i}$ is manifestly saturated in $\mathscr{B}_{d}$ (being the base extension of a subspace of $\left.\mathbb{D}_{\text {cris }}(V(m))\right)$, and together with (a), this proves (b). For 
part (c), we note that $\mathscr{A}_{d} \cap \mathscr{B}_{i-1}=\mathscr{A}_{i-1}$, so the given map is well-defined and injective; to show that the annihilator is as claimed, we must check that

$$
\left(\frac{t}{\pi}\right)^{m-s_{i}} \mathscr{B}_{d} \subseteq \mathscr{B}_{i-1}+\mathscr{A}_{d}
$$

which is equivalent to Lemma 2.6.

We now pass from the "additive" to the "multiplicative" situation. Let us define $\widetilde{A}_{i}=\bigoplus_{s=1}^{p-1}(1+\pi)^{s} \varphi\left(\mathscr{A}_{i}\right)$, and similarly for $\widetilde{\mathscr{P}}_{i}$. Note that these are $\Gamma$-stable, since $\Gamma$ and $\varphi$ commute. Moreover, the action of $\Gamma$ on $\widetilde{\mathscr{P}}_{d}$ clearly extends to an action of the ring $\mathscr{H}(\Gamma)$, which is continuous with respect to the Fréchet topologies of $\mathscr{H}(\Gamma)$ and $\widetilde{\mathscr{S}}_{d}$. As the submodules $\widetilde{\mathscr{B}}_{i}$ and $\widetilde{\mathscr{A}}_{i}$ are all clearly closed and $\Gamma$-invariant, they also inherit a Fréchet topology and a continuous action of $\mathscr{H}(\Gamma)$.

Remark 2.8. Note that we can define an operator $\psi: \mathscr{B}_{d} \rightarrow \mathscr{B}_{d}$ which is $\varphi^{-1}$ on $\mathbb{D}_{\text {cris }}(V)$ and is $\mathbb{B}_{\text {rig, } \mathbb{Q}_{p}}^{+}$-semilinear (for the usual definition of $\psi$ acting on $\mathbb{B}_{\text {rig, } \mathbb{Q}_{p}}^{+}$). Then $\widetilde{\mathscr{A}}_{i}=\left(\varphi^{*} \mathscr{A}_{i}\right)^{\psi=0}$, where $\varphi^{*} \mathscr{A}_{i}$ is the $\mathbb{B}_{\text {rig, } \mathbb{Q}_{p}}^{+}$-submodule of $\mathscr{B}_{i}$ generated by $\varphi\left(\mathscr{A}_{i}\right)$. Clearly we have $\varphi^{*}\left(\mathscr{B}_{i}\right)=\mathscr{B}_{i}$ for all $i$, and $\widetilde{\mathscr{B}}_{i}=\left(\varphi^{*} \mathscr{B}_{i}\right)^{\psi=0}=\mathscr{S}_{i}^{\psi=0}$.

Lemma 2.9. For each $i=1, \ldots, d$, these spaces have the following properties:

(a) $\tilde{\mathscr{A}}_{i} \subseteq \widetilde{\mathscr{B}}_{i}$.

(b) $\tilde{\mathscr{A}}_{d} \cap \widetilde{\mathscr{B}}_{i}=\tilde{\mathscr{A}}_{i}$.

(c) The quotient $\widetilde{\mathscr{P}}_{d} /\left(\widetilde{\mathscr{P}}_{i-1}+\widetilde{\mathscr{A}}_{d}\right)$ is annihilated by $\mathfrak{n}_{m-s_{i}}$.

(d) The quotient $\widetilde{\mathscr{P}}_{i} /\left(\widetilde{\mathscr{P}}_{i-1}+\widetilde{\mathscr{A}}_{i}\right)$ is cyclic as a $\mathscr{H}(\Gamma)$-module; it is generated by $(1+\pi) \varphi\left(v_{i}\right)$, and its annihilator is $\mathfrak{n}_{m-s_{i}}$.

Proof. Parts (a) and (b) are clear from the corresponding statements for the spaces $\mathscr{A}_{i}$ and $\mathscr{B}_{i}$. For part (c), we note that $\mathscr{B}_{d} / \mathscr{B}_{i-1}$ is isomorphic as a $(\varphi, \Gamma)$-module over $\mathbb{B}_{\text {rig, }, \mathbb{Q}_{p}}^{+}$to the tensor product

$$
\mathbb{B}_{\text {rig, } \mathbb{Q}_{p}}^{+} \otimes_{\mathbb{Q}_{p}}\left(Y_{d} / Y_{i-1}\right)
$$

with $\Gamma$ acting trivially on the latter factor and the $\varphi$-action multiplied by $p^{-m}$. By Proposition 1.6, we have

$$
\mathfrak{n}_{k} \cdot\left(\mathscr{B}_{d} / \mathscr{B}_{i-1}\right)^{\psi=0}=\left(\left(\frac{t}{\varphi(\pi)}\right)^{k} \mathscr{B}_{d} / \mathscr{B}_{i-1}\right)^{\psi=0} .
$$

Since $\mathscr{B}_{d} /\left(\mathscr{B}_{i-1}+\mathscr{A}_{d}\right)$ is annihilated by $\left(\frac{t}{\pi}\right)^{m-s_{i}}$, we deduce that $\mathscr{B}_{d} /\left(\mathscr{B}_{i-1}+\varphi^{*} \mathscr{A}_{d}\right)$ is annihilated by

$$
\left(\frac{t}{\varphi(\pi)}\right)^{m-s_{i}}
$$

Hence, by $(*), \widetilde{\mathscr{P}}_{d} /\left(\widetilde{\mathscr{P}}_{i-1}+\widetilde{\mathscr{A}}_{d}\right)$ is annihilated by the ideal $\mathfrak{n}_{m-s_{i}}$ of $\mathscr{H}\left(\Gamma_{1}\right)$. 
Similarly, $\mathscr{B}_{i} /\left(\mathscr{B}_{i-1}+\mathscr{A}_{i}\right)$ has the single elementary divisor

$$
\left(\frac{t}{\pi}\right)^{m-s_{i}}
$$

(by applying Proposition 2.3 to $\mathscr{X}_{i} / \mathscr{X}_{i-1}$, which is a $(\varphi, \Gamma)$-module over $\mathbb{B}_{\text {rig, } \mathbb{Q}_{p}}^{+}$ by Proposition 2.4(e)). Hence we deduce that $\mathfrak{n}_{m-s_{i}}$ is the exact annihilator of the corresponding $\mathscr{H}(\Gamma)$-module $\widetilde{\mathscr{B}}_{i} /\left(\widetilde{\mathscr{P}}_{i-1}+\widetilde{\mathscr{A}}_{i}\right)$.

We are now in a position to complete the proof of Theorem A.

Theorem 2.10 (Theorem A). Let $W$ be any E-linear crystalline representation of $G_{\mathbb{Q}_{p}}$ with non-negative Hodge-Tate weights $r_{1} \leq \cdots \leq r_{d}$. Suppose that there exists a finite extension $F$ of $E$ such that $V \otimes_{E} F$ admits a non-critical refinement. Then the $E \otimes \mathscr{H}(\Gamma)$-elementary divisors of the quotient

$$
\left(\mathbb{B}_{\text {rig, }, \mathbb{Q}_{p}}^{+}\right)^{\psi=0} \otimes \mathbb{D}_{\text {cris }}(W) /\left(\varphi^{*} \mathbb{N}_{\text {rig }}(W)\right)^{\psi=0}
$$

are $\left[\mathfrak{n}_{r_{1}} ; \ldots ; \mathfrak{n}_{r_{d}}\right]$.

Proof. Let us choose an $m$ such that $V=W(-m)$ is positive. Then the Hodge-Tate weights of $V$ are $-s_{1} \geq \cdots \geq-s_{d}$, where $s_{i}=m-r_{d+1-i} \geq 0$. Suppose first that $V$ admits a non-critical refinement. Choosing such a refinement, we may argue as above to deduce that the $E \otimes \mathscr{H}(\Gamma)$-module

$$
M=\left(\mathbb{B}_{\text {rig }, \mathbb{Q}_{p}}^{+}\right)^{\psi=0} \otimes \mathbb{D}_{\text {cris }}(W) /\left(\varphi^{*} \mathbb{N}_{\text {rig }}(W)\right)^{\psi=0}=\mathscr{B}_{d} / \mathscr{A}_{d}
$$

has a filtration by $E \otimes_{\mathbb{Q}_{p}} \mathscr{H}(\Gamma)$-modules $M_{i}=\mathscr{B}_{i} / \mathscr{A}_{i}$ where $M_{i} / M_{i-1}$ is cyclic with annihilator $\mathfrak{n}_{m-s_{i}}$, and $\mathfrak{n}_{m-s_{i}}$ annihilates $M / M_{i-1}$. So for each character $\eta$ of $\Delta$, the module $M^{\eta}$ is an $\mathscr{H}\left(\Gamma_{1}\right)$-module of the type covered by Corollary 1.12 . This gives the result in this special case.

If $V$ only admits a non-critical refinement after extending scalars to an extension $F / E$, then we may consider the representation $V \otimes_{E} F$ and apply the above argument to this representation. It is clear that if $M$ is any $E \otimes \mathscr{H}(\Gamma)$-module, then the elementary divisors of $F \otimes_{E} M$ as a $F \otimes \mathscr{H}(\Gamma)$-module are the base extensions of the elementary divisors of $M$; by uniqueness, this gives the proposition.

We now briefly explain how $\varphi^{*} \mathbb{N}_{\text {rig }}(V)$ is related to the Wach module $\mathbb{N}(V)$ considered in our earlier work. Note that $\mathcal{H}(\Gamma)$ and $\varphi\left(\mathbb{B}_{\text {rig, } \mathbb{Q}_{p}}^{+}\right)$are both FréchetStein algebras in the sense of [Schneider and Teitelbaum 2003] (by Theorem 5.1 of that reference); hence any finite-rank free module over either one of these algebras has a canonical topology, and a submodule of such a module is finitely generated if and only if it is closed in this topology (Corollary 3.4(ii) of [op. cit.]). Moreover, $\left(\mathbb{B}_{\text {rig, } \mathbb{Q}_{p}}^{+}\right)^{\psi=0}=\bigoplus_{i=1}^{p-1}(1+\pi)^{i} \varphi\left(\mathbb{B}_{\text {rig, } \mathbb{Q}_{p}}^{+}\right)$is a free module over $\varphi\left(\mathbb{B}_{\text {rig, } \mathbb{Q}_{p}}^{+}\right)$of rank $p-1$. 
Proposition 2.11. There is an isomorphism

$$
\left(\varphi^{*} \mathbb{N}_{\text {rig }}(V)\right)^{\psi=0} \cong \mathscr{H}(\Gamma) \otimes_{\Lambda_{\mathbb{Q}_{p}}(\Gamma)}\left(\varphi^{*} \mathbb{N}(V)\right)^{\psi=0} .
$$

Proof. We first note that $\left(\varphi^{*} \mathbb{N}_{\text {rig }}(V)\right)^{\psi=0}=\bigoplus_{i=1}^{p-1}(1+\pi)^{i} \varphi\left(\mathbb{N}_{\text {rig }}(V)\right)$ is a finitely generated $\varphi\left(\mathbb{B}_{\text {rig, } \mathbb{Q}_{p}}^{+}\right)$-submodule of $\mathbb{D}_{\text {cris }}(V) \otimes_{\mathbb{Q}_{p}}\left(\mathbb{B}_{\text {rig, } \mathbb{Q}_{p}}^{+}\right)^{\psi=0}$. Hence it is closed in the canonical Fréchet topology of the latter space. It is also $\Gamma$-stable. Since the Mellin transform is a topological isomorphism between $\left(\mathbb{B}_{\text {rig, } \mathbb{Q}_{p}}^{+}\right)^{\psi=0}$ and $\mathscr{H}(\Gamma)$, we see that $\left(\varphi^{*} \mathbb{N}_{\text {rig }}(V)\right)^{\psi=0}$ is a closed $\Gamma$-stable subspace of a finite-rank free $\mathscr{H}(\Gamma)$ module; hence the action of $\Gamma$ extends to a (continuous) action of $\mathscr{H}(\Gamma)$. So there is a natural embedding of $\mathscr{H}(\Gamma) \otimes_{\Lambda_{\mathbb{Q}_{p}}(\Gamma)}\left(\varphi^{*} \mathbb{N}(V)\right)^{\psi=0}$ into $\left(\varphi^{*} \mathbb{N}_{\text {rig }}(V)\right)^{\psi=0}$.

The image of this embedding is a $\mathscr{H}(\Gamma)$-submodule, which is finitely generated, since $\left(\varphi^{*} \mathbb{N}(V)\right)^{\psi=0}$ is finitely generated as a $\Lambda_{E}$-module [Lei et al. 2010, Theorem 3.5]. So it is closed. On the other hand, the image contains $\left(\varphi^{*} \mathbb{N}(V)\right)^{\psi=0}$. Since we evidently have

$$
\left(\varphi^{*} \mathbb{N}_{\mathrm{rig}}(V)\right)^{\psi=0}=\bigoplus_{i=1}^{p-1}(1+\pi)^{i} \varphi\left(\mathbb{N}_{\mathrm{rig}}(V)\right)
$$

and $\varphi\left(\mathbb{N}_{\text {rig }}(V)\right)=\varphi\left(\mathbb{B}_{\text {rig, } \mathbb{Q}_{p}}^{+}\right) \otimes_{\varphi\left(\mathbb{B}_{\mathbb{Q}_{p}}^{+}\right)} \varphi(\mathbb{N}(V))$, it follows that

$$
\begin{aligned}
\left(\varphi^{*} \mathbb{N}_{\text {rig }}(V)\right)^{\psi=0} & =\varphi\left(\mathbb{B}_{\text {rig, }, \mathbb{Q}_{p}}^{+}\right) \otimes_{\varphi\left(\mathbb{B}_{\mathbb{Q}_{p}}^{+}\right)}\left(\bigoplus_{i=1}^{p-1}(1+\pi)^{i} \varphi(\mathbb{N}(V))\right) \\
& =\varphi\left(\mathbb{B}_{\text {rig, } \mathbb{Q}_{p}}^{+}\right) \otimes_{\varphi\left(\mathbb{B}_{\mathbb{Q}_{p}}^{+}\right)}\left(\varphi^{*} \mathbb{N}(V)\right)^{\psi=0} .
\end{aligned}
$$

Since $\varphi\left(\mathbb{B}_{\mathbb{Q}_{p}}^{+}\right)$is dense in $\varphi\left(\mathbb{B}_{\text {rig, } \mathbb{Q}_{p}}^{+}\right)$, it follows now that $\left(\varphi^{*} \mathbb{N}(V)\right)^{\psi=0}$ is dense in $\left(\varphi^{*} \mathbb{N}_{\text {rig }}(V)\right)^{\psi=0}$. Thus the image of $\mathscr{H}(\Gamma) \otimes_{\Lambda_{\mathbb{Q}_{p}}(\Gamma)}\left(\varphi^{*} \mathbb{N}(V)\right)^{\psi=0}$ in $\left(\varphi^{*} \mathbb{N}_{\text {rig }}(V)\right)^{\psi=0}$ is both dense and closed; hence it is everything.

We recall the following result from our previous work:

Theorem 2.12 ([Lei et al. 2010, Lemma 3.15]). $\left(\varphi^{*} \mathbb{N}(V)\right)^{\psi=0}$ is a free $\Lambda_{E}(\Gamma)$ module of rank $d$. More specifically, for any basis $v_{1}, \ldots, v_{d}$ of $\mathbb{D}_{\text {cris }}(V)$, there exists a $E \otimes \mathbb{B}_{\mathbb{Q}_{p}}^{+}$-basis $n_{1}, \ldots, n_{d}$ of $\mathbb{N}(V)$ such that $n_{i}=v_{i} \bmod \pi$ and $(1+$ $\pi) \varphi\left(n_{1}\right), \ldots,(1+\pi) \varphi\left(n_{d}\right)$ form a $\Lambda_{E}(\Gamma)$-basis of $\left(\varphi^{*} \mathbb{N}(V)\right)^{\psi=0}$.

Combining this with Proposition 2.11, the following corollary is immediate:

Corollary 2.13. $\left(\varphi^{*} \mathbb{N}_{\text {rig }}(V)\right)^{\psi=0}$ is a free $E \otimes \mathscr{H}(\Gamma)$-module of rank $d$. More specifically, for any basis $v_{1}, \ldots, v_{d}$ of $\mathbb{D}_{\text {cris }}(V)$, there exists a $E \otimes \mathbb{B}_{\text {rig, } \mathbb{Q}_{p}}^{+}{ }^{-b a s i s}$ $n_{1}, \ldots, n_{d}$ of $\mathbb{N}_{\text {rig }}(V)$ such that $n_{i}=v_{i} \bmod \pi$ and $(1+\pi) \varphi\left(n_{1}\right), \ldots,(1+\pi) \varphi\left(n_{d}\right)$ form a $E \otimes \mathscr{H}(\Gamma)$-basis of $\left(\varphi^{*} \mathbb{N}_{\text {rig }}(V)\right)^{\psi=0}$. 
Remark 2.14. We conjecture that for any $E \otimes \mathbb{B}_{\text {rig, } \mathbb{Q}_{p}}^{+}$-basis $m_{1}, \ldots, m_{d}$ of $\mathbb{N}_{\text {rig }}(V)$, the vectors $(1+\pi) \varphi\left(m_{i}\right)$ are a $E \otimes \mathscr{H}(\Gamma)$-basis of $\left(\varphi^{*} \mathbb{N}_{\text {rig }}(V)\right)^{\psi=0}$, and similarly for $\mathbb{N}(V)$; but we do not know a proof of this statement.

\section{The construction of Coleman maps}

3A. Coleman maps and the Perrin-Riou p-adic regulator. Let $E$ be a finite extension of $\mathbb{Q}_{p}$. Let $V$ be a $d$-dimensional $E$-linear representation of $G_{\mathbb{Q}_{p}}$ with non-negative Hodge-Tate weights $r_{1} \leq r_{2} \leq \cdots \leq r_{d}$. We assume that $V$ has no quotient isomorphic to the trivial representation. Let $T$ be a $\mathscr{G}_{\mathbb{Q}_{p}}$-stable $\mathscr{O}_{E}$-lattice in $V$. Under these assumptions, there is a canonical isomorphism of $\Lambda_{\mathscr{C}_{E}}(\Gamma)$ modules

$$
h_{\mathrm{Iw}}^{1}: \mathbb{N}(T)^{\psi=1} \stackrel{\cong}{\longrightarrow} H_{\mathrm{IW}}^{1}\left(\mathbb{Q}_{p}, T\right) .
$$

by [Berger 2003, Theorem A.3]. Moreover, since the Hodge-Tate weights of $V$ are non-negative, we have $\mathbb{N}(T) \subseteq \varphi^{*} \mathbb{N}(T)$ by Lemma 1.7. Hence there is a welldefined map $1-\varphi: \mathbb{N}(T) \rightarrow \varphi^{*} \mathbb{N}(T)$, which maps $\mathbb{N}(T)^{\psi=1}$ to $\left(\varphi^{*} \mathbb{N}(T)\right)^{\psi=0}$.

As we recalled above, [Lei et al. 2010, Theorem 3.5] (due to Laurent Berger) shows that for some basis $n_{1}, \ldots, n_{d}$ of $\mathbb{N}(T)$ as an $\mathbb{O}_{E} \otimes \mathbb{A}_{\mathbb{Q}_{p}}^{+}$-module, the vectors $(1+\pi) \varphi\left(n_{1}\right), \ldots,(1+\pi) \varphi\left(n_{d}\right)$ form a basis of $\left(\varphi^{*} \mathbb{N}(T)\right)^{\psi=0}$ as a $\Lambda_{\mathscr{C}_{E}}(\Gamma)$-module. This basis gives an isomorphism

$$
\mathfrak{J}:\left(\varphi^{*} \mathbb{N}(T)\right)^{\psi=0} \stackrel{\cong}{\longrightarrow} \Lambda_{\mathscr{O}_{E}}(\Gamma)^{\oplus d}
$$

(the Iwasawa transform), and we define the Coleman map

$$
\underline{\mathrm{Col}}=\left(\underline{\mathrm{Col}}_{i}\right)_{i=1}^{d}: \mathbb{N}(T)^{\psi=1} \rightarrow \Lambda_{\mathfrak{O}_{E}}(\Gamma)^{\oplus d}
$$

as the composition $\mathfrak{J} \circ(1-\varphi)$.

Remark 3.1. This direct definition of the Coleman map is equivalent to that given in our earlier work, but applies to any representation with non-negative HodgeTate weights, rather than starting with a positive representation and twisting by the sum of its Hodge-Tate weights as in [Lei et al. 2010].

Let $v_{1}, \ldots, v_{d}$ be a basis of $\mathbb{D}_{\text {cris }}(V)$, so $(1+\pi) \otimes v_{1}, \ldots,(1+\pi) \otimes v_{d}$ are a basis of $\left(\mathbb{B}_{\text {rig, } \mathbb{Q}_{p}}^{+}\right)^{\psi=0} \otimes \mathbb{D}_{\text {cris }}(V)$ as an $\mathscr{H}(\Gamma)$-module; and let $n_{1}, \ldots, n_{d}$ be a basis of $\mathbb{N}(V)$ lifting $v_{1}, \ldots, v_{d}$ as in Theorem 2.12. Then there exists a unique $d \times d$ matrix $\underline{M}$ with entries in $\mathscr{H}(\Gamma)$ such that

$$
\left(\begin{array}{c}
(1+\pi) \varphi\left(n_{1}\right) \\
\vdots \\
(1+\pi) \varphi\left(n_{d}\right)
\end{array}\right)=\underline{M} \cdot\left(\begin{array}{c}
(1+\pi) \otimes v_{1} \\
\vdots \\
(1+\pi) \otimes v_{d}
\end{array}\right) .
$$


In fact $\underline{M}$ is defined over $\mathscr{H}\left(\Gamma_{1}\right)$, since the $n_{i}$ lie in $(1+\pi) \varphi(\mathbb{N}(V)) \subseteq(1+$ $\pi) \varphi\left(\mathbb{B}_{\text {rig, } \mathbb{Q}_{p}}^{+}\right) \otimes \mathbb{D}_{\text {cris }}(V)$. By Theorem 2.10 , we know that the elementary divisors of $\underline{M}$ are $\mathfrak{n}_{r_{1}}, \ldots, \mathfrak{n}_{r_{d}}$.

Corollary 3.2. Up to a unit, $\operatorname{det}(\underline{M})$ is equal to $\prod_{i=1}^{d} \mathfrak{n}_{r_{i}}$.

We can write the Coleman map $\underline{\mathrm{Col}}$ in terms of $\underline{M}$ as follows:

Lemma 3.3. For $x \in \mathbb{N}(T)^{\psi=1}$, we have

$$
(1-\varphi)(x)=\underline{\operatorname{Col}}(x) \cdot \underline{M} \cdot\left(\begin{array}{c}
(1+\pi) \otimes v_{1} \\
\vdots \\
(1+\pi) \otimes v_{d}
\end{array}\right) .
$$

Proof. We have by definition

$$
(1-\varphi) x=\underline{\operatorname{Col}}(x) \cdot\left(\begin{array}{c}
(1+\pi) \varphi\left(n_{1}\right) \\
\vdots \\
(1+\pi) \varphi\left(n_{d}\right)
\end{array}\right) .
$$

Therefore, we are done on combining this with (1).

Definition 3.4. The Perrin-Riou p-adic regulator $\mathscr{L}_{V}$ for $V$ is defined to be the $\Lambda_{E}(\Gamma)$-homomorphism

$$
\left(\mathfrak{M}^{-1} \otimes 1\right) \circ(1-\varphi) \circ\left(h_{\mathrm{Iw}, V}^{1}\right)^{-1}: H_{\mathrm{Iw}}^{1}\left(\mathbb{Q}_{p}, V\right) \longrightarrow \mathscr{H}(\Gamma) \otimes \mathbb{D}_{\text {cris }}(V) .
$$

Using the isomorphism $h_{\mathrm{Iw}, V}^{1}: \mathbb{N}(V)^{\psi=1} \rightarrow H_{\mathrm{IW}}^{1}\left(\mathbb{Q}_{p}, V\right)$, we can thus rewrite Lemma 3.3 as

$$
\mathscr{L}_{V}(z)=\left(\underline{\mathrm{Col}} \circ\left(h_{\mathrm{Iw}, V}^{1}\right)^{-1}\right)(z) \cdot \underline{M} \cdot\left(\begin{array}{c}
v_{1} \\
\vdots \\
v_{d}
\end{array}\right) .
$$

\section{Images of the Coleman maps}

Let $\eta$ be a character on $\Delta$. In this section, we study the image of $\underline{\operatorname{Col}}^{\eta}\left(\mathbb{N}(V)^{\psi=1}\right)$ as a subset of $\Lambda_{E}\left(\Gamma_{1}\right)^{\oplus d}$ for a crystalline representation $V$ of dimension $d$ with non-negative Hodge-Tate weights. We then consider the projection of this image, giving a description of $\operatorname{Im}\left(\underline{\mathrm{Col}}_{i}^{\eta}\right)$ for $i=1, \ldots, d$.

4A. Preliminary results on $\Lambda_{\boldsymbol{E}}\left(\Gamma_{1}\right)$-modules. Recall that we identify $\Lambda_{E}\left(\Gamma_{1}\right)$ with the power series ring $E \otimes \mathcal{O}_{E} \llbracket X \rrbracket$ by identifying $\gamma-1$ with $X$. Therefore, if $F \in \Lambda_{E}\left(\Gamma_{1}\right)$ and $x$ is an element of the maximal ideal of $E,\left.F\right|_{X=x} \in E$. 
Lemma 4.1. Let $V$ be an E-subspace of $E^{d}$ with codimension $n$. For a fixed element $x$ of the maximal ideal of $E$, we define the $\Lambda_{E}\left(\Gamma_{1}\right)$-module

$$
S=\left\{\left(F_{1}, \ldots, F_{d}\right) \in \Lambda_{E}\left(\Gamma_{1}\right)^{\oplus d}:\left(F_{1}(x), \ldots, F_{d}(x)\right) \in V\right\} .
$$

Then, $S$ is free of rank $d$ over $\Lambda_{E}\left(\Gamma_{1}\right)$ and $\operatorname{det}(S)=(X-x)^{n}$.

Proof. Let $v_{1}, \ldots, v_{d}$ be a basis of $E$ such that $\sum_{i=1}^{d} e_{i} v_{i} \in V$ if and only if $e_{i}=0$ for all $i>d-n$. On multiplying elementary matrices in $\mathrm{GL}_{d}(E)$ if necessary, we may assume that $S$ is of the form

$$
\begin{aligned}
S & =\left\{\left(F_{1}, \ldots, F_{d}\right) \in \Lambda_{E}\left(\Gamma_{1}\right)^{\oplus d}: F_{d-n+1}(x)=\cdots=F_{d}(x)=0\right\} \\
& =\Lambda_{E}\left(\Gamma_{1}\right)^{\oplus(d-n)} \oplus\left((X-x) \Lambda_{E}\left(\Gamma_{1}\right)\right)^{\oplus n},
\end{aligned}
$$

so we are done.

Proposition 4.2. Let $I=\left\{x_{0}, \ldots, x_{m}\right\}$ be a subset of the maximal ideal of $E$. For each $i=0, \ldots, m$, let $V_{i}$ be an $E$-subspace of $E^{\oplus d}$ with codimension $n_{i}$. Define

$$
S=\left\{\left(F_{1}, \ldots, F_{d}\right) \in \Lambda_{E}\left(\Gamma_{1}\right)^{\oplus d}:\left(F_{1}\left(x_{i}\right), \ldots, F_{d}\left(x_{i}\right)\right) \in V_{i}, i=0, \ldots, m\right\},
$$

then $S$ is free of rank $d$ over $\Lambda_{E}\left(\Gamma_{1}\right)$, and $\operatorname{det}(S)=\prod_{i=0}^{m}\left(X-x_{i}\right)^{n_{i}}$.

Proof. We prove the result by induction on $m$. The case $m=0$ is just Lemma 4.1.

Assume that $m>0$ and let

$$
S^{\prime}=\left\{\left(F_{1}, \ldots, F_{d}\right) \in \Lambda_{E}\left(\Gamma_{1}\right)^{\oplus d}:\left(F_{1}\left(x_{i}\right), \ldots, F_{d}\left(x_{i}\right)\right) \in V_{i}, i=0, \ldots, m-1\right\} .
$$

By induction, $S^{\prime}$ is free of rank $d$ over $\Lambda_{E}\left(\Gamma_{1}\right)$ and $\operatorname{det}\left(S^{\prime}\right)=\prod_{i=0}^{m-1}\left(X-x_{i}\right)^{n_{i}}$. Let $F^{(i)}=\left(F_{1}^{(i)}, \ldots, F_{d}^{(i)}\right), i=1, \ldots, d$, be a $\Lambda_{E}\left(\Gamma_{1}\right)$-basis of $S^{\prime}$. Write $\mathscr{F}_{m}$ for the $d \times d$ matrix with entries $F_{j}^{(i)}\left(x_{m}\right)$. As $X-x_{m}$ does not divide $\operatorname{det}\left(F_{j}^{(i)}\right)$, we have $\mathscr{F}_{m} \in \mathrm{GL}_{d}(E)$. Define

$$
S^{\prime \prime}=\left\{\left(G_{1}, \ldots, G_{d}\right) \in \Lambda_{E}\left(\Gamma_{1}\right)^{\oplus d}:\left(G_{1}\left(x_{m}\right), \ldots, G_{d}\left(x_{m}\right)\right) \in V_{m} \mathscr{F}_{m}^{-1}\right\} .
$$

By Lemma 4.1, $S^{\prime \prime}$ is free of rank $d$ over $\Lambda_{E}\left(\Gamma_{1}\right)$ and $\operatorname{det}\left(S^{\prime \prime}\right)=\left(X-x_{m}\right)^{n_{m}}$. Say, $\left(G_{1}^{(k)}, \ldots, G_{d}^{(k)}\right), k=1, \ldots, d$, is a basis.

For $\left(G_{1}, \ldots, G_{d}\right) \in \Lambda_{E}\left(\Gamma_{1}\right)^{\oplus d}$, we have $\sum_{i=1}^{d} G_{i} F^{(i)} \in S^{\prime} \subset S$ by definition. It is easy to check that $\sum_{i=1}^{d} G_{i} F^{(i)} \in S$ if and only if $\left(G_{1}, \ldots, G_{d}\right) \in S^{\prime \prime}$. Therefore, a basis for $S$ is given by the row vectors of $\left(G_{i}^{(k)}\right)\left(F_{j}^{(i)}\right)$ and $\operatorname{det}(S)=\operatorname{det}\left(S^{\prime}\right) \operatorname{det}\left(S^{\prime \prime}\right)$. Hence, we are done.

Lemma 4.3. If $S$ is a $\Lambda_{E}\left(\Gamma_{1}\right)$-module as in the statement of Proposition 4.2, then the image of a projection from $S$ into $\Lambda_{E}\left(\Gamma_{1}\right)$ is of the form $\prod_{i \in J}\left(X-x_{i}\right) \Lambda_{E}\left(\Gamma_{1}\right)$ where $J$ is some subset of $\{0, \ldots, m\}$. 
Proof. We consider the first projection $\mathrm{pr}_{1}:\left(F_{1}, \ldots, F_{d}\right) \mapsto F_{1}$. Let

$$
J=\left\{i \in[0, m]:\left(e_{1}, \ldots, e_{d}\right) \in V_{i} \Rightarrow e_{1}=0\right\} .
$$

It is clear that $\operatorname{Im}\left(\mathrm{pr}_{1}\right) \subset \prod_{i \in J}\left(X-x_{i}\right) \Lambda_{E}\left(\Gamma_{1}\right)$. It remains to show that

$$
\prod_{i \in J}\left(X-x_{i}\right) \in \operatorname{Im}\left(\mathrm{pr}_{1}\right)
$$

By definition, for each $i \notin J$, there exist $e_{k}^{(i)} \in E, k=2, \ldots, d$, such that

$$
\left(\prod_{j \in J}\left(x_{i}-x_{j}\right), e_{2}^{(i)}, \ldots, e_{d}^{(i)}\right) \in V_{i} .
$$

Similarly, take any $\left(0, e_{2}^{(i)}, \ldots, e_{d}^{(i)}\right) \in V_{i}$ for $i \in J$. There exist polynomials $F_{k}$ over $E$ such that $F_{k}\left(x_{i}\right)=e_{j}^{(i)}$ for $k=2, \ldots, d$ and $i=0, \ldots, m$. It is then clear that

$$
\left(\prod_{i \in J}\left(X-x_{i}\right), F_{2}, \ldots, F_{d}\right) \in S .
$$

Hence we are done.

4B. On the image of the Perrin-Riou p-adic regulator. with Hodge-Tate weights $-r_{d} \leq-r_{d-1} \leq \cdots \leq-r_{1} \leq 0$. As in Section 3A, fix bases $n_{1}, \ldots, n_{d}$ and $v_{1}, \ldots, v_{d}$ of $\mathbb{N}(\mathscr{T})$ and $\mathbb{D}_{\text {cris }}(\mathscr{V})$, respectively, such that $v_{i}=n_{i} \bmod \pi$. For the rest of this paper, we make the following assumption.

$\mathscr{G}_{\mathbb{Q}_{p}}$ with non-negative Hodge-Tate weights $m-r_{1} \geq \cdots \geq m-r_{d} \geq 0$. Moreover, our assumption on the eigenvalues of $\varphi$ implies that $V$ has no quotient isomorphic to $E$.

Let $V$ be a $d$-dimensional $E$-linear crystalline representation of $\mathscr{G}_{\mathbb{Q}_{p}}$ with nonnegative Hodge-Tate weights $r_{1} \leq \cdots \leq r_{d}$.

Definition 4.4. For an integer $i \geq 0$, we write

$$
n_{i}=\operatorname{dim}_{E} \operatorname{Fil}^{-i} \mathbb{D}_{\text {cris }}(V)=\#\left\{j: r_{j} \leq i\right\} .
$$

We make the following assumption:

Assumption 4.5. The eigenvalues of $\varphi$ on $\mathbb{D}_{\text {cris }}(V)$ are not integer powers of $p$.

Recall from [Perrin-Riou 1994] that we have the exponential map

$$
\Omega_{V, r_{d}}:\left(\mathbb{B}_{\text {rig, } \mathbb{Q}_{p}}^{+}\right)^{\psi=0} \otimes \mathbb{D}_{\text {cris }}(V) \rightarrow \mathscr{H}(\Gamma) \otimes H_{\mathrm{Iw}}^{1}\left(\mathbb{Q}_{p}, V\right) .
$$

The Perrin-Riou $p$-adic regulator is related to $\Omega_{V, r_{d}}$ via the following equation. 
Theorem 4.6. As maps on $H_{\mathrm{Iw}}^{1}\left(\mathbb{Q}_{p}, V\right)$, we have

$$
\mathscr{L}_{V}=\left(\mathfrak{M}^{-1} \otimes 1\right)\left(\prod_{i=0}^{r_{d}-1} \ell_{i}\right)\left(\Omega_{V, r_{d}}\right)^{-1} .
$$

Proof. By definition, this is the same as saying

$$
(1-\varphi) \circ\left(h_{\mathrm{Iw}, V}^{1}\right)^{-1}=\left(\prod_{i=0}^{r_{d}-1} \ell_{i}\right)\left(\Omega_{V, r_{d}}\right)^{-1},
$$

which is just a rewrite of [Berger 2003, Theorem II.13].

Corollary 4.7. We have

$$
\operatorname{det}\left(\mathscr{L}_{V}\right)=\prod_{i=0}^{r_{d}-1}\left(\ell_{i}\right)^{d-n_{i}} .
$$

Proof. The $\delta(V)$-conjecture (see [Perrin-Riou 1994, Conjecture 3.4.7]) predicts that

$$
\operatorname{det}\left(\Omega_{V, r_{d}}\right)=\prod_{i \leq r_{d}-1}\left(\ell_{i}\right)^{n_{i}}
$$

As pointed out in Proposition 3.6.7 of the same work, this conjecture is a consequence of Perrin-Riou's explicit reciprocity law, labeled "Conjecture (Réc)" in [op. cit.], and proved in [Colmez 1998, théorème IX.4.5]. Therefore, Theorem 4.6 implies that

$$
\operatorname{det}\left(\mathscr{L}_{V}\right)=\left(\prod_{i=0}^{r_{d}-1}\left(\ell_{i}\right)^{d}\right)\left(\prod_{i \leq r_{d}-1}\left(\ell_{i}\right)^{-n_{i}}\right),
$$

which finishes the proof, since $n_{i}=0$ for $i<0$.

Let $z \in H_{\mathrm{Iw}}^{1}\left(\mathbb{Q}_{p}, V\right)$. Then $\mathscr{L}_{V}(z) \in \mathscr{H}(\Gamma) \otimes_{\mathbb{Q}_{p}} \mathbb{D}_{\text {cris }}(V)$, so we can apply to $\mathscr{L}_{V}(z)$ any character on $\Gamma$ to obtain an element in $\mathbb{D}_{\text {cris }}(V)$. The following proposition studies elements obtained in this way when we choose characters of a specific kind. Recall that we denote by $\chi$ the cyclotomic character, and by $\chi_{0}$ the restriction of $\chi$ to $\Delta$.

Proposition 4.8. Let $z \in H_{\mathrm{Iw}}^{1}\left(\mathbb{Q}_{p}, V\right)$. Then for any integer $0 \leq i \leq r_{d}-1$ and any Dirichlet character $\delta$ of conductor $p^{n}>1$, we have

$$
(1-\varphi)^{-1}\left(1-p^{-1} \varphi^{-1}\right) \chi^{i}\left(\mathscr{L}_{V}(z) \otimes t^{i} e_{-i}\right) \in \operatorname{Fil}^{0} \mathbb{D}_{\text {cris }}(V(-i)) ;
$$

and

$$
\varphi^{-n}\left(\chi^{i} \delta\left(\mathscr{L}_{V}(z) \otimes t^{i} e_{-i}\right)\right) \in \mathbb{Q}_{p, n} \otimes \operatorname{Fil}^{0} \mathbb{D}_{\text {cris }}(V(-i))
$$


Proof. We write [ , ] for the pairing

$$
\mathbb{D}_{\text {cris }}(V(-i)) \times \mathbb{D}_{\text {cris }}\left(V^{*}(1+i)\right) \longrightarrow \mathbb{D}_{\text {cris }}(E(1))=E \cdot t^{-1} e_{1} .
$$

The orthogonal complement of $\mathrm{Fil}^{0} \mathbb{D}_{\text {cris }}(V(-i))$ under [ , ] is $\mathrm{Fil}^{0}\left(V^{*}(1+i)\right)$. Let $x \in \operatorname{Fil}^{0} \mathbb{D}_{\text {cris }}\left(V^{*}(1+i)\right)$ and $x^{\prime}=(1-\varphi)\left(1-p^{-1} \varphi^{-1}\right)^{-1} x$, and write $x_{-i}^{\prime}$ for $x^{\prime} \otimes t^{i} e_{-i}$. Then

$$
\begin{aligned}
{\left[(1-\varphi)^{-1}\left(1-p^{-1} \varphi^{-1}\right) \chi^{i}\left(\mathscr{L}_{V}(z) \otimes t^{i} e_{-i}\right), x\right] } & =\left[\chi^{i}\left(\mathscr{L}_{V}(z) \otimes t^{i} e_{-i}\right), x^{\prime}\right] \\
& =\chi^{i}\left[\mathscr{L}_{V}(z), x_{-i}^{\prime}\right],
\end{aligned}
$$

where the first equality follows from the observation that $1-\varphi$ and $1-p^{-1} \varphi^{-1}$ are adjoint to each other under the pairing [ , ].

We extend [ , ] to a pairing on

$$
\mathscr{H}(\Gamma) \otimes_{\mathbb{Q}_{p}} \mathbb{D}_{\text {cris }}(V) \times \mathscr{H}(\Gamma) \otimes_{\mathbb{Q}_{p}} \mathbb{D}_{\text {cris }}\left(V^{*}(1)\right) \longrightarrow E \otimes_{\mathbb{Q}_{p}} \mathscr{H}(\Gamma)
$$

in the natural way. By Perrin-Riou's explicit reciprocity law (see previous proof) and Theorem 4.6, we have

$$
\left[\mathscr{L}_{V}(z), x_{-i}^{\prime}\right]=(-1)^{r_{d}-1}\left\langle\left(\prod_{j=0}^{r_{d}-1} \ell_{j}\right) z, \Omega_{V^{*}(1), 1-r_{d}}\left((1+\pi) \otimes x_{-i}^{\prime}\right)\right\rangle
$$

where $\langle$,$\rangle denotes the pairing$

$$
\left(\mathscr{H}(\Gamma) \otimes H_{\mathrm{Iw}}^{1}\left(\mathbb{Q}_{p}, V\right)\right) \times\left(\mathscr{H}(\Gamma) \otimes H_{\mathrm{Iw}}^{1}\left(\mathbb{Q}_{p}, V^{*}(1)\right)\right) \longrightarrow E \otimes \mathscr{H}(\Gamma)
$$

as defined in [Perrin-Riou 1994, § 3.6]. By [Perrin-Riou 1994, Lemme 3.6.1(i)], the right-hand side of (5) in fact equals

$$
\left\langle z,\left(\prod_{j=0}^{r_{d}-1} \ell_{-j}\right) \Omega_{V^{*}(1), 1-r_{d}}\left((1+\pi) \otimes x_{-i}^{\prime}\right)\right\rangle=\left\langle z, \Omega_{V^{*}(1), 1}\left((1+\pi) \otimes x_{-i}^{\prime}\right)\right\rangle .
$$

By an abuse of notation, we let Tw denote the twist map on the $H_{\mathrm{Iw}}^{1}$ 's as well as the map on $\mathscr{H}(\Gamma)$ that sends any $g \in \Gamma$ to $\chi(g) g$. We have

$$
\left\langle\operatorname{Tw}^{-i}(x), \operatorname{Tw}^{i}(y)\right\rangle=\operatorname{Tw}^{i}\langle x, y\rangle
$$

for any $x$ and $y$ by [Perrin-Riou 1994, Lemme 3.6.1(ii)]. Therefore, by combining (5) and (6), $\chi^{i}\left[\mathscr{L}_{V}(z), x_{-i}^{\prime}\right]$ is equal to the projection of

$$
\left\langle\operatorname{Tw}^{-i}(z), \operatorname{Tw}^{i}\left(\Omega_{V^{*}(1), 1}\left((1+\pi) \otimes x_{-i}^{\prime}\right)\right)\right\rangle
$$

into $E$. The projection of $\operatorname{Tw}^{i}\left(\Omega_{V^{*}(1), 1}\left((1+\pi) \otimes x_{-i}^{\prime}\right)\right)$ into $H^{1}\left(\mathbb{Q}_{p}, V^{*}(1+i)\right)$ at the origin is equal to a scalar multiple of

$$
\exp _{\mathbb{Q}_{p}, V^{*}(1+i)}\left(\left(1-p^{-1} \varphi^{-1}\right)(1-\varphi)^{-1}\left(x^{\prime}\right)\right)
$$


(see for example [Lei et al. 2010, Proposition 3.19]). But

$$
\left(1-p^{-1} \varphi^{-1}\right)(1-\varphi)^{-1}\left(x^{\prime}\right)=x \in \operatorname{Fil}^{0} \mathbb{D}_{\text {cris }}\left(V^{*}(1+i)\right)
$$

by definition. Therefore, as $\exp _{\mathbb{Q}_{p}, V^{*}(1+i)}$ vanishes on $\mathrm{Fil}^{0} \mathbb{D}_{\text {cris }}\left(V^{*}(1+i)\right)$ by construction, it follows that

$$
\exp _{\mathbb{Q}_{p}, V^{*}(1+i)}\left((1-\varphi)^{-1}\left(1-p^{-1} \varphi^{-1}\right)\left(x^{\prime}\right)\right)=0
$$

and hence that

$$
\left[(1-\varphi)^{-1}\left(1-p^{-1} \varphi^{-1}\right) \chi^{i}\left(\mathscr{L}_{V}(z) \otimes t^{i} e_{-i}\right), x\right]=\chi^{i}\left[\mathscr{L}_{V}(z), x_{-i}^{\prime}\right]=0 .
$$

This implies (3), and (4) can be proved similarly.

For any character $\eta$ of $\Delta$ and an integer $0 \leq i \leq r_{d}-1$, define

$$
V_{i, \eta}= \begin{cases}\left(1-p^{i} \varphi\right)\left(1-p^{-1-i} \varphi^{-1}\right)^{-1} \mathrm{Fil}^{-i} \mathbb{D}_{\text {cris }}(V) & \text { if } \chi_{0}^{i}=\eta, \\ \varphi\left(\mathrm{Fil}^{-i} \mathbb{D}_{\text {cris }}(V)\right) & \text { otherwise. }\end{cases}
$$

Note that $V_{i, \eta}$ is a subspace of $\mathbb{D}_{\text {cris }}(V)$ of the same dimension as Fil ${ }^{-i} \mathbb{D}_{\text {cris }}(V)$.

Corollary 4.9. If $\eta$ is a character on $\Delta$, then

$$
\left\{\chi^{i} \chi_{0}^{-i} \eta\left(e_{\eta} \mathscr{L}_{V}(z)\right): z \in H_{\mathrm{Iw}}^{1}\left(\mathbb{Q}_{p}, V\right)\right\} \subset V_{i, \eta} .
$$

Proof. Note that $\mathrm{Fil}^{-i} \mathbb{D}_{\text {cris }}(V)=\mathrm{Fil}^{0} \mathbb{D}_{\text {cris }}(V(-i)) \otimes t^{-i} e_{i}$. Therefore, if $\chi_{0}^{i}=\eta$, the result follows from (3) and the fact that $\varphi\left(t^{i} e_{-i}\right)=p^{i} t^{i} e_{-i}$. Assume otherwise. Since $\left.\chi^{i} \chi_{0}^{-i} \eta\right|_{\Delta}=\eta$, we have $\chi^{i} \chi_{0}^{-i} \eta\left(e_{\eta} \mathscr{L}_{V}(z)\right)=\chi^{i} \chi_{0}^{-i} \eta\left(\mathscr{L}_{V}(z)\right)$. Hence, (4) implies that

$$
\varphi^{-1}\left(\chi^{i} \chi_{0}^{-i} \eta\left(e_{\eta} \mathscr{L}_{V}(z) \otimes t^{i} e_{-i}\right)\right) \in \mathbb{Q}_{p}\left(\mu_{p}\right) \otimes \operatorname{Fil}^{0} \mathbb{D}_{\text {cris }}(V(-i)) .
$$

But

$$
\chi^{i} \chi_{0}^{-i} \eta\left(e_{\eta} \mathscr{L}_{V}(z) \otimes t^{i} e_{-i}\right)=\left.\mathscr{L}_{V}(z)^{\eta}\right|_{X=\chi(\gamma)^{i}-1} \otimes t^{i} e_{-i}
$$

in fact lies inside $\mathbb{D}_{\text {cris }}(V(-i))$. Hence,

$$
\varphi^{-1}\left(\chi^{i} \chi_{0}^{-i} \eta\left(e_{\eta} \mathscr{L}_{V}(z) \otimes t^{i} e_{-i}\right)\right) \in \operatorname{Fil}^{0} \mathbb{D}_{\text {cris }}(V(-i))=\operatorname{Fil}^{-i} \mathbb{D}_{\text {cris }}(V) \otimes t^{i} e_{-i}
$$

and we are done on applying $\varphi$ to both sides.

Corollary 4.10. If $\eta$ is a character on $\Delta$, then

$$
\left\{\left.\mathscr{L}_{V}(z)^{\eta}\right|_{X=\chi(\gamma)^{i}-1}: z \in H_{\mathrm{IW}}^{1}\left(\mathbb{Q}_{p}, V\right)\right\} \subset V_{i, \eta} .
$$

Proof. This is immediate from Corollary 4.9, since

$$
\left.\mathscr{L}_{V}(z)^{\eta}\right|_{X=\chi(\gamma)^{i}-1}=\chi^{i} \chi_{0}^{-i} \eta\left(e_{\eta} \mathscr{L}_{V}(z)\right) .
$$


4C. Images of the Coleman maps. We now fix a character $\eta: \Delta \rightarrow \mathbb{Z}_{p}^{\times}$. Let $v_{1}, \ldots, v_{d}$ be a basis of $\mathbb{D}_{\text {cris }}(V)$ and $n_{1}, \ldots, n_{d}$ a basis of $\mathbb{N}(V)$ lifting $v_{1}, \ldots, v_{d}$ as in Theorem 2.12. We consider the image of the Coleman map defined with respect to this basis as in Section 3.

Proposition 4.11. The image of the map

$$
{\mathrm{Col}^{\eta}}^{\eta} \mathbb{N}(V)^{\psi=1} \longrightarrow \Lambda_{E}\left(\Gamma_{1}\right)^{\oplus d}
$$

lies inside a $\Lambda_{E}\left(\Gamma_{1}\right)$-submodule $S$ as described in the statement of Proposition 4.2 with

$$
I=\left\{x_{i}=\chi(\gamma)^{i}-1: 0 \leq i \leq r_{d}-1\right\} \quad \text { and } \quad V_{i}=V_{i, \eta},
$$

which is an E-vector space of the same (co-)dimension as $\mathrm{Fil}^{-i} \mathbb{D}_{\text {cris }}(V)$.

Proof. Recall from (2) that

$$
\mathscr{L}_{V}=\left(\underline{\mathrm{Col}} \circ h_{\mathrm{Iw}, V}^{1}\right) \underline{M}\left(\begin{array}{c}
v_{1} \\
\vdots \\
v_{d}
\end{array}\right)
$$

where $\underline{M}$ is as defined in (1). Note that $\underline{M}^{\eta}=\underline{M}$ for any character $\eta$ of $\Delta$, since $\underline{M}$ is defined over $\mathcal{H}\left(\Gamma_{1}\right)$. Moreover, Corollary 3.2 implies that $X-\chi(\gamma)^{i}+1$ does not divide $\operatorname{det}(\underline{M})$, so $\left.\underline{M}\right|_{X=\chi(\gamma)^{i}-1} \in \mathrm{GL}_{d}(E)$. Therefore, we are done by Corollary 4.10.

Theorem 4.12. Equality holds in Proposition 4.11.

Proof. Write $S$ for the basis matrix of the $\Lambda_{E}\left(\Gamma_{1}\right)$-submodule of $\Lambda_{E}\left(\Gamma_{1}\right)^{\oplus d}$ described in the statement of Proposition 4.11. Then, Proposition 4.2 says that

$$
\operatorname{det}(S)=\prod_{i=0}^{r_{d}-1}\left(X-\chi(\gamma)^{i}+1\right)^{d-n_{i}}
$$

But

$$
\operatorname{det}(\underline{M})=\prod_{j=1}^{d}\left(\prod_{i=0}^{r_{j}-1} \frac{\ell_{i}}{X-\chi(\gamma)^{i}+1}\right)=\prod_{i=0}^{r_{d}-1}\left(\frac{\ell_{i}}{X-\chi(\gamma)^{i}+1}\right)^{d-n_{i}},
$$

since $n_{i}=\#\left\{j: r_{j} \leq i\right\}$, as noted above. Hence, Corollary 4.7 implies that

$$
\operatorname{det}\left(\mathscr{L}_{V}\right)=\operatorname{det}(\underline{M}) \operatorname{det}(S)
$$

and we are done.

We can summarize the above results via the following short exact sequence: 
Corollary 4.13. Suppose that no eigenvalue of $\varphi$ on $\mathbb{D}_{\text {cris }}(V)$ lies in $p^{\mathbb{Z}}$. Then for each character $\eta$ of $\Delta$, there is a short exact sequence of $\mathcal{H}\left(\Gamma_{1}\right)$-modules

$$
0 \longrightarrow \mathbb{N}(V)^{\psi=1, \eta} \stackrel{1-\varphi}{\longrightarrow}\left(\varphi^{*} \mathbb{N}(V)\right)^{\psi=0, \eta} \stackrel{A_{\eta}}{\longrightarrow} \bigoplus_{i=0}^{r_{d}-1}\left(\mathbb{D}_{\text {cris }}(V) / V_{i, \eta}\right)\left(\chi^{i} \chi_{0}^{-i} \eta\right) \longrightarrow 0
$$

Here the map $A_{\eta}$ equals $\bigoplus_{i}\left(1 \otimes A_{\eta, i}\right)$, where $A_{\eta, i}$ is the natural reduction map $\mathscr{H}(\Gamma) \rightarrow \mathbb{Q}_{p}\left(\chi^{i} \chi_{0}^{-i} \eta\right)$ obtained by quotienting out by the ideal $\left(X+1-\chi(\gamma)^{i}\right) \cdot e_{\eta}$.

Remark 4.14. The short exact sequence in Corollary 4.13 can be seen as an analogue of Perrin-Riou's exact sequence (see [Perrin-Riou 1994, §2.2])

$$
\begin{aligned}
0 \longrightarrow \bigoplus_{i=0}^{r_{d}} t^{i} \mathbb{D}_{\text {cris }}(V)^{\varphi=p^{-i}} \longrightarrow\left(\mathbb{B}_{\text {rig, } \mathbb{Q}_{p}}^{+} \otimes \mathbb{D}_{\text {cris }}(V)\right)^{\psi=1} \\
\stackrel{\varphi-1}{\longrightarrow}\left(\mathbb{B}_{\text {rig, } \mathbb{Q}_{p}}^{+}\right)^{\psi=0} \otimes \mathbb{D}_{\text {cris }}(V) \longrightarrow \bigoplus_{i=0}^{r_{d}}\left(\frac{\mathbb{D}_{\text {cris }}(V)}{1-p^{i} \varphi}\right)(i) \longrightarrow 0 .
\end{aligned}
$$

In particular, the injectivity of the first map in our sequence follows from PerrinRiou's sequence, whose first term vanishes in view of our assumption on $V$.

We can now prove Theorem $\mathrm{C}$.

Corollary 4.15. For $i=1, \ldots, d$, we have

$$
\operatorname{Im}\left(\underline{\operatorname{Col}}_{i}^{\eta}\right)=\prod_{j \in I_{i}^{\eta}}\left(X-\chi(\gamma)^{j}+1\right) \Lambda_{E}\left(\Gamma_{1}\right)
$$

for some $I_{i}^{\eta} \subset\left\{0, \ldots, r_{d}-1\right\}$.

Proof. This follows immediately from Lemma 4.3.

We can also use this argument to determine the elementary divisors of the cokernel of the map $\mathscr{L}_{V}$, refining the result of Corollary 4.7.

Theorem 4.16. The elementary divisors of the $\mathscr{H}(\Gamma)$-module quotient

$$
\frac{\mathscr{H}(\Gamma) \otimes_{\mathbb{Q}_{p}} \mathbb{D}_{\text {cris }}(V)}{\mathcal{H}(\Gamma) \otimes_{\Lambda_{\mathbb{Q}_{p}}(\Gamma)} \operatorname{Im}\left(\mathscr{L}_{V}\right)}
$$

are $\left[\lambda_{r_{1}} ; \ldots ; \lambda_{r_{d}}\right]$, where $\lambda_{k}=\ell_{0} \ell_{1} \ldots \ell_{k-1}$.

Proof. We know that the matrix of $\mathscr{L}_{V}$ is equal to $M \cdot S$, where $M$ and $S$ have elementary divisors that are coprime. Hence the elementary divisors of the product matrix are the products of the elementary divisors, which gives the above formula. 


\section{The Coleman maps for modular forms}

In this section, we fix a modular form $f$ as in Section 1C6. We pick bases $n_{1}, n_{2}$ of $\mathbb{N}\left(T_{\bar{f}}\right)$ and $\bar{v}_{1}, \bar{v}_{2}$ of $\mathbb{D}_{\text {cris }}\left(V_{\bar{f}}\right)$ as in [Lei et al. 2010, Section 3.3]. Let $V=V_{\bar{f}}(k-1)$, which has Hodge-Tate weights 0 and $k-1$. We consider the Coleman maps $\mathrm{Col}_{1}^{\eta}$ and $\underline{\mathrm{Col}}_{2}^{\eta}$ defined on $\mathbb{N}(V)^{\psi=1}$ where $\eta$ is a fixed character on $\Delta$. As a special case for Theorem 4.12 and Corollary 4.15, we have the following result.

Proposition 5.1. There exist 1-dimensional E-subspaces $V_{i}$ of $E^{2}$ for $0 \leq i<k-1$ such that

$$
\operatorname{Im}\left(\underline{\mathrm{Col}}^{\eta}\right)=\left\{(F, G) \in \Lambda_{E}\left(\Gamma_{1}\right):\left(F\left(\chi^{i}(\gamma)-1\right), G\left(\chi^{i}(\gamma)-1\right)\right) \in V_{i}\right\} .
$$

Moreover, for $l=1,2$, we have

$$
\operatorname{Im}\left(\underline{\operatorname{Col}}_{l}^{\eta}\right)=\prod_{j \in I_{l}^{\eta}}\left(X-\chi^{j}(\gamma)+1\right) \Lambda_{E}\left(\Gamma_{1}\right)
$$

for some $I_{l}^{\eta} \subset\{0, \ldots, k-2\}$ with $I_{1}^{\eta}$ and $I_{2}^{\eta}$ disjoint.

Proof. For $0 \leq j \leq k-2$, Fil $^{-j} \mathbb{D}_{\text {cris }}(V)$ is of dimension 1 over $E$. Hence the first part of the proposition by Theorem 4.12. The second part of the proposition follows by putting

$$
I_{1}^{\eta}=\left\{i: V_{i}=0 \oplus E\right\} \quad \text { and } \quad I_{2}^{\eta}=\left\{i: V_{i}=E \oplus 0\right\} .
$$

Remark 5.2. Note that the second part of the proposition is a slightly stronger version of Corollary 4.15.

Corollary 5.3. In particular, there exist nonzero elements $r_{i} \in E$ for $i \in I_{3}^{\eta}:=$ $\{0, \ldots, k-2\} \backslash\left(I_{1}^{\eta} \cup I_{2}^{\eta}\right)$ such that

$$
\operatorname{Im}\left(\underline{\mathrm{Col}}^{\eta}\right)=\left\{\begin{array}{l|l}
(F, G) \in \Lambda_{E}\left(\Gamma_{1}\right) & \begin{array}{l}
F\left(u^{i}-1\right)=0 \text { if } i \in I_{1}^{\eta} \\
G\left(u^{i}-1\right)=0 \text { if } i \in I_{2}^{\eta} \\
F\left(u^{i}-1\right)=r_{i} G\left(u^{i}-1\right) \text { if } i \in I_{3}^{\eta}
\end{array}
\end{array}\right\}
$$

where $u=\chi(\gamma)$.

The aim of this section is to study the set above in more detail.

5A. Some explicit linear relations. Recall from [Lei et al. 2010, proof of Proposition 3.22] that the maps $\mathscr{L}_{1}$ and $\mathscr{L}_{2}$ as defined in Section 1 C6 satisfy

$$
\mathscr{L}_{V}(z)=-\mathscr{L}_{2}(z) \bar{v}_{1, k-1}+\mathscr{L}_{1}(z) \bar{v}_{2, k-1}
$$

for any $z \in H_{\mathrm{IW}}^{1}\left(\mathbb{Q}_{p}, V\right)$. Therefore, Corollary 4.9 says that $\mathscr{L}_{1}(z)$ and $\mathscr{L}_{2}(z)$ satisfy some linear relations when evaluated at $\chi^{j} \delta$ for $0 \leq j \leq k-2$ and $\delta$ some character on $\Delta$. We now make these relations explicit. First we recall that we have: 
Lemma 5.4. Let $j, n \geq 0$ be integers and $i \in\{1,2\}$. For $z \in H_{\mathrm{Iw}}^{1}\left(\mathbb{Q}_{p}, V\right)$, we write $z_{-j, n}$ for the image of $z$ under

$$
H_{\mathrm{Iw}}^{1}\left(\mathbb{Q}_{p}, V\right) \rightarrow H_{\mathrm{Iw}}^{1}\left(\mathbb{Q}_{p}, V(-j)\right) \rightarrow H^{1}\left(\mathbb{Q}_{p, n}, V(-j)\right)
$$

where the first map is the twist map $(-1)^{j} \mathrm{Tw}_{j}$ and the second map is the projection. Then, we have

$$
\chi^{j}\left(\mathscr{L}_{i}(z)\right)=j !\left[(1-\varphi)^{-1}\left(1-p^{-1} \varphi^{-1}\right) v_{i, j+1}, \exp _{\mathbb{Q}_{p}, V(j)}^{*}\left(z_{-j, 0}\right)\right] .
$$

If $\delta$ is a character of $G_{n}$ which does not factor through $G_{n-1}$ with $n \geq 1$, then

$$
\chi^{j} \delta\left(\mathscr{L}_{i}(z)\right)=\frac{j !}{\tau\left(\delta^{-1}\right)} \sum_{\sigma \in G_{n}} \delta^{-1}(\sigma)\left[\varphi^{-n}\left(v_{i, j+1}\right), \exp _{\mathbb{Q}_{p, 1}, V(j)}^{*}\left(z_{-j, n}^{\sigma}\right)\right]
$$

where $\tau$ denotes the Gauss sum.

Proof. See, for example, [Lei 2011, Lemma 3.5 and (4)].

Lemma 5.5. If $0 \leq j \leq k-2$ and $\delta$ is a nontrivial character on $\Delta$, then

$$
\chi^{j} \delta\left(\mathscr{L}_{2}(z)\right)=0 .
$$

Proof. On putting $n=1$ in (9), we have

$$
\chi^{j} \delta\left(\mathscr{L}_{2}(z)\right)=\frac{j !}{\tau\left(\delta^{-1}\right)} \sum_{\sigma \in \Delta} \delta^{-1}(\sigma)\left[\varphi^{-1}\left(v_{2, j+1}\right), \exp _{\mathbb{Q}_{p, 1}, V(j)}^{*}\left(z_{-j, 1}^{\sigma}\right)\right] .
$$

But $v_{2}=p^{1-k} \varphi\left(v_{1}\right)$, so

$$
\varphi^{-1}\left(v_{2, j+1}\right) \in E \cdot v_{1, j+1}=\operatorname{Fil}^{0} \mathbb{D}_{\text {cris }}\left(V_{f}(j+1)\right) .
$$

Therefore, we have

$$
\left[\varphi^{-1}\left(v_{2, j+1}\right), \exp _{\mathbb{Q}_{p, 1}, V(j)}^{*}\left(z^{\sigma}\right)\right]=0
$$

for all $\sigma \in \Delta$ and we are done.

Lemma 5.6. If $\varphi^{2}+a \varphi+b=0$, then

$$
(1-\varphi)^{-1}\left(1-p^{-1} \varphi^{-1}\right)=\frac{(1+a+p b) \varphi+a(1+a+p b)+b(p-1)}{p b(1+a+b)} .
$$

Proof. We can write $\varphi^{2}+a \varphi+b=0, \varphi^{2}-1+a(\varphi-1)=-1-a-b$, and $(1-\varphi)(\varphi+1+a)=1+a+b$. Therefore,

$$
(1-\varphi)^{-1}=\frac{\varphi+1+a}{1+a+b} .
$$

Similarly, we have

$$
\varphi^{-1}=-\frac{\varphi+a}{b}
$$

The result then follows from explicit calculation. 
Corollary 5.7. For $0 \leq j \leq k-2$, we have

$$
\left(-a_{p}+p^{j+1}+p^{k-1-j}\right) \chi^{j}\left(\mathscr{L}_{2}(z)\right)=(p-1) \chi^{j}\left(\mathscr{L}_{1}(z)\right) .
$$

Proof. On $\mathbb{D}_{\text {cris }}\left(V_{\bar{f}}(k-1-j)\right), \varphi$ satisfies

$$
\varphi^{2}-a_{p} p^{-k+1+j} \varphi+p^{-k+1+2 j}=0,
$$

as we assume $\varepsilon(p)=1$. Let

$$
\begin{aligned}
u & =1-a_{p} p^{-k+1+j}+p^{-k+2+2 j}, \\
u^{\prime} & =-a_{p} p^{-k+1+j} u+p^{-k+1+2 j}(p-1) .
\end{aligned}
$$

Then, Proposition 4.8 and Lemma 5.6 imply that

$$
\left(u \varphi+u^{\prime}\right) \chi^{j}\left(-\mathscr{L}_{2}(z) \bar{v}_{1, k-1-j}+\mathscr{L}_{1}(z) \bar{v}_{2, k-1-j}\right)
$$

lies in $\mathrm{Fil}^{0} \mathbb{D}_{\text {cris }}\left(V_{\bar{f}}(k-1-j)\right)=E v_{1, k-1-j}$. On writing this expression as a linear combination of $v_{1, k-1-j}$ and $v_{2, k-1-j}$, the coefficient of the latter turns out to be

$$
-p^{j} u \chi^{j}\left(\mathscr{L}_{2}(z)\right)+\left(u^{\prime}+a_{p} p^{-k+1+j} u\right) \chi^{r}\left(\mathscr{L}_{1}(z)\right),
$$

which must be zero, hence the result.

Remark 5.8. The coefficient $-a_{p}+p^{j+1}+p^{k-1-j}$ is nonzero by the Weil bound.

Recall from [Lei et al. 2010, (32)] that we have

$$
\left(\begin{array}{ll}
-\mathscr{L}_{2} & \mathscr{L}_{1}
\end{array}\right)=\left(\underline{\mathrm{Col}} \circ h_{\mathrm{Iw}, V}^{1} \underline{M} .\right.
$$

By [Lei et al. 2010, proof of Proposition 3.28 and Theorem 5.4], we have

$$
\left.\underline{M}\right|_{X=0}=A_{\varphi}^{T}=\left(\begin{array}{rc}
0 & p^{k-1} \\
-1 & a_{p}
\end{array}\right) .
$$

Therefore, the relations for $j=0$ are given by

$$
\begin{array}{ccc}
\left.\left(-a_{p}+1+p^{k-2}\right) \underline{\operatorname{Col}}_{2}(x)^{\Delta}\right|_{X=0}=\left.p^{k-2}(p-1) \underline{\operatorname{Col}}_{1}(x)^{\Delta}\right|_{X=0} & & \text { if } \eta=1, \\
\left.\underline{\operatorname{Col}}_{2}(x)^{\eta}\right|_{X=0}=0 & & \text { if } \eta \neq 1 .
\end{array}
$$

In particular, for the case $k=2$, we have the following analogue of [Kurihara and Pollack 2007, Proposition 1.2].

Proposition 5.9. If $k=2$, the trivial isotypical component of the Coleman maps give a short exact sequence

$$
0 \longrightarrow H_{\mathrm{Iw}}^{1}\left(\mathbb{Q}_{p}, V\right) \stackrel{\mathrm{Col}^{\Delta}}{\longrightarrow} \Lambda_{E}\left(\Gamma_{1}\right) \oplus \Lambda_{E}\left(\Gamma_{1}\right) \stackrel{\rho}{\longrightarrow} \mathbb{Q}_{p} \longrightarrow 0,
$$

where $\rho$ is defined by

$$
\rho(g(X), h(X))=\left(2-a_{p}\right) g(0)-(p-1) h(0) .
$$


5B. Integral structure of the images. We now describe the integral structure of $\operatorname{Im}\left(\underline{\mathrm{Col}}_{i}\right)^{\eta}$. Under the notation of Corollary 5.3, we define

$$
X_{i}^{\eta}=\prod_{j \in I_{i}^{\eta}}\left(X-\chi(\gamma)^{j}+1\right) .
$$

Then, we have:

Theorem 5.10. For $i=1,2$, let $X_{i}^{\eta}$ be as defined above. Then

$$
\underline{\mathrm{Col}}_{i}\left(\mathbb{D}\left(T_{\bar{f}}(k-1)\right)^{\psi=1}\right)^{\eta} \subset X_{i}^{\eta} \Lambda_{\bigcirc_{E}}\left(\Gamma_{1}\right) .
$$

Moreover, $X_{i}^{\eta} \Lambda_{\widehat{O}_{E}}\left(\Gamma_{1}\right) / \underline{\operatorname{Col}}_{i}\left(\mathbb{D}\left(T_{\bar{f}}(k-1)\right)^{\psi=1}\right)^{\eta}$ is pseudo-null.

Proof. Let

$$
X_{k}=\prod_{j=0}^{k-2}\left(X-\chi(\gamma)^{j}+1\right) .
$$

Note that the proof of Proposition 4.11 in [Lei et al. 2010] is true integrally. We therefore have

$$
\left(\varphi^{k-1}(\pi) \varphi^{*} \mathbb{N}\left(T_{\bar{f}}(k-1)\right)\right)^{\psi=0} \subset(1-\varphi) \mathbb{N}\left(T_{\bar{f}}(k-1)\right)^{\psi=1} .
$$

This implies that $X_{k} \in \operatorname{Im}\left(\underline{\mathrm{Col}}_{i}\right)$ for $i=1,2$. Hence, we have the following inclusions:

$$
X_{k} \Lambda_{\bigcirc_{E}}\left(\Gamma_{1}\right) \subset \underline{\operatorname{Col}}_{i}\left(\mathbb{D}\left(T_{\bar{f}}(k-1)\right)^{\psi=1}\right)^{\eta} \subset X_{i}^{\eta} \Lambda_{\mathscr{C}_{E}}\left(\Gamma_{1}\right)
$$

for $i=1,2$. Since $X_{k}$ is not divisible by $\varpi_{E}$, the quotient

$$
X_{i}^{\eta} \Lambda_{\mathscr{O}_{E}}\left(\Gamma_{1}\right) / X_{k} \Lambda_{\mathscr{O}_{E}}\left(\Gamma_{1}\right)
$$

is a free $\mathbb{O}_{E}$-module of finite rank. Moreover, for a coset representative, $x$ say, it follows from Corollary 4.15 that there exists an integer $n$ such that

$$
\varpi_{E}^{n} x \in \underline{\operatorname{Col}}_{i}\left(\mathbb{D}\left(T_{\bar{f}}(k-1)\right)^{\psi=1}\right)^{\eta} .
$$

Therefore, $\underline{\operatorname{Col}}_{i}\left(\mathbb{D}\left(T_{\bar{f}}(k-1)\right)^{\psi=1}\right)^{\eta}$ is of finite index in $X_{i}^{\eta} \Lambda_{\mathscr{O}_{E}}\left(\Gamma_{1}\right)$.

5C. Surjectivity via a change of basis. Unfortunately, we do not have an explicit description of the sets $I_{i}^{\eta}$ given by Corollary 5.3. However, this can be resolved by choosing a different basis:

Proposition 5.11. Let $S$ be a subset of $\Lambda_{E}\left(\Gamma_{1}\right)^{\oplus 2}$ as defined in Corollary 5.3. Then, there exists $A \in \mathrm{GL}\left(2, O_{E}\right)$ such that $S A=S^{\prime}$ for some $S^{\prime}$ which is of the form

$$
\left\{(F, G) \in \Lambda_{E}\left(\Gamma_{1}\right)^{\oplus 2}: F\left(u^{i}-1\right)=r_{i}^{\prime} G\left(u^{i}-1\right), 0 \leq i \leq k-2\right\}
$$

for some nonzero elements $r_{i}^{\prime} \in E$. 
Proof. Let $e_{1}, e_{2} \in \mathbb{O}_{E}$ be nonzero elements such that $e_{1} e_{2} \neq 1$, then

$$
\left(\begin{array}{cc}
1 & e_{2} \\
e_{1} & 1
\end{array}\right) \in \mathrm{GL}\left(2, \mathrm{O}_{E}\right)
$$

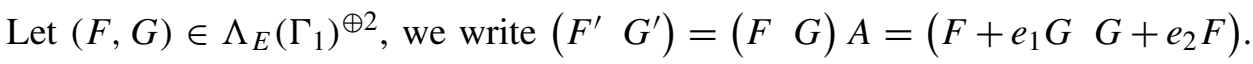
We have

$$
\begin{aligned}
F\left(u^{i}-1\right)=0 & \Longleftrightarrow F^{\prime}\left(u^{i}-1\right)=e_{1} G^{\prime}\left(u^{i}-1\right) ; \\
G\left(u^{i}-1\right)=0 & \Longleftrightarrow G^{\prime}\left(u^{i}-1\right)=e_{2} F^{\prime}\left(u^{i}-1\right) ; \\
F\left(u^{i}-1\right)=r_{i} G\left(u^{i}-1\right) & \Longleftrightarrow\left(e_{2} r_{i}+1\right) F^{\prime}\left(u^{i}-1\right)=\left(e_{1}+r_{i}\right) G^{\prime}\left(u^{i}-1\right) .
\end{aligned}
$$

Therefore, we are done on choosing $e_{2} \neq-r_{i}^{-1}$ and $e_{1} \neq-r_{i}$ for all $i \in I_{3}^{\eta}$.

Remark 5.12. In the construction of the Coleman maps, replacing $\left(\begin{array}{l}n_{1} \\ n_{2}\end{array}\right)$ by $A\left(\begin{array}{l}n_{1} \\ n_{2}\end{array}\right)$, where $A \in \mathrm{GL}\left(2, \mathrm{O}_{E}\right)$, is equivalent to replacing $\underline{M}$ by $A \underline{M}$.

Therefore, on multiplying $\underline{M}$ by an appropriate matrix in $\operatorname{GL}\left(2, O_{E}\right)$ on the left, we can make both Coleman maps surjective (though we cannot assume $\left.\underline{M}\right|_{X=0}=$ $A_{\varphi}^{T}$ any more). By Proposition 5.11, we deduce

Theorem 5.13. There exists a basis of $\mathbb{N}\left(T_{\bar{f}}\right)$ such that the corresponding Coleman maps have the following properties:

$$
\Lambda_{\bigcirc_{E}}\left(\Gamma_{1}\right) / \underline{\operatorname{Col}}_{i}\left(\mathbb{D}\left(T_{\bar{f}}(k-1)\right)^{\psi=1}\right)^{\eta}
$$

is pseudo-null for $i=1,2$.

\section{Acknowledgements}

We are very grateful to Bernadette Perrin-Riou for giving us some unpublished notes about her $p$-adic regulator; and to the anonymous referee, for numerous helpful comments and corrections.

\section{References}

[Amice and Vélu 1975] Y. Amice and J. Vélu, "Distributions $p$-adiques associées aux séries de Hecke", pp. 119-131 in Journées Arithmétiques de Bordeaux (Bordeaux, 1974), Astérisque 24-25, Soc. Math. France, Paris, 1975. MR 51 \#12709 Zbl 0332.14010

[Bellaïche and Chenevier 2009] J. Bellaïche and G. Chenevier, Families of Galois representations and Selmer groups, Astérisque 324, 2009. MR 2011m:11105 Zbl 1192.11035

[Berger 2002] L. Berger, "Représentations p-adiques et équations différentielles", Invent. Math. 148:2 (2002), 219-284. MR 2004a:14022 Zbl 1113.14016

[Berger 2003] L. Berger, "Bloch and Kato's exponential map: three explicit formulas", pp. 99-129 in Kazuya Kato's fiftieth birthday, edited by S. Bloch et al., Documenta Mathematica, Bielefeld, 2003. Collection appeared as an unnumbered extra volume of Doc. Math. (2003). MR 2005f:11268 Zbl 1064.11077 
[Berger 2004] L. Berger, "Limites de représentations cristallines", Compos. Math. 140:6 (2004), 1473-1498. MR 2098398 (2006c:11138) Zbl 1071.11067

[Berger et al. 2004] L. Berger, H. Li, and H. J. Zhu, "Construction of some families of 2-dimensional crystalline representations”, Math. Ann. 329:2 (2004), 365-377. MR 2005k:11104 Zbl 1085.11028

[Cherbonnier and Colmez 1999] F. Cherbonnier and P. Colmez, "Théorie d'Iwasawa des représentations $p$-adiques d'un corps local”, J. Amer. Math. Soc. 12:1 (1999), 241-268. MR 99g:11141 Zbl 0933.11056

[Colmez 1998] P. Colmez, "Théorie d'Iwasawa des représentations de de Rham d'un corps local", Ann. of Math. (2) 148:2 (1998), 485-571. MR 2000f:11077 Zbl 0928.11045

[Deligne 1971] P. Deligne, "Formes modulaires et représentations $\ell$-adiques", pp. 139-172 (exposé 355) in Séminaire Bourbaki, 1968/69, Lecture Notes in Mathematics 179, Springer, Berlin, 1971. Zbl 0206.49901

[Kato 2004] K. Kato, " $p$-adic Hodge theory and values of zeta functions of modular forms", pp. 117-290 in Cohomologies p-adiques et applications arithmétiques, III, edited by P. Berthelot et al., Astérisque 295, Société Mathématique de France, Paris, 2004. MR 2006b:11051 Zbl 1142.11336

[Kurihara and Pollack 2007] M. Kurihara and R. Pollack, "Two $p$-adic $L$-functions and rational points on elliptic curves with supersingular reduction", pp. 300-332 in L-functions and Galois representations (Durham, 2004), edited by D. Burns et al., London Math. Soc. Lecture Note Ser. 320, Cambridge Univ. Press, 2007. MR 2009g:11069 Zbl 1148.11029

[Lang 2002] S. Lang, Algebra, 3rd ed., Graduate Texts in Mathematics 211, Springer, New York, 2002. MR 2003e:00003 Zbl 0984.00001

[Lei 2011] A. Lei, "Iwasawa theory for modular forms at supersingular primes", Compos. Math. 147:3 (2011), 803-838. MR 2012e:11186 Zbl 1234.11148

[Lei et al. 2010] A. Lei, D. Loeffler, and S. L. Zerbes, "Wach modules and Iwasawa theory for modular forms", Asian J. Math. 14:4 (2010), 475-528. MR 2774276 Zbl 05878707

[Loeffler and Zerbes 2012] D. Loeffler and S. L. Zerbes, "Wach modules and critical slope $p$-adic L-functions", J. Reine Angew. Math. (2012).

[Milne 1994] J. S. Milne, "Motives over finite fields", pp. 401-459 in Motives (Seattle, 1991), edited by U. Jannsen et al., Proc. Sympos. Pure Math. 55, Amer. Math. Soc., Providence, RI, 1994. MR 95g:11053 Zbl 0811.14018

[Perrin-Riou 1994] B. Perrin-Riou, “Théorie d'Iwasawa des représentations $p$-adiques sur un corps local”, Invent. Math. 115:1 (1994), 81-161. MR 95c:11082 Zbl 0838.11071

[Perrin-Riou 1995] B. Perrin-Riou, Fonctions L p-adiques des représentations p-adiques, Astérisque 229, Soc. Mat. de France, Paris, 1995. MR 96e:11062 Zbl 0845.11040

[Perrin-Riou 2001] B. Perrin-Riou, Théorie d'Iwasawa des représentations p-adiques semi-stables, Mém. Soc. Math. Fr. (N.S.) 84, Soc. Mat. de France, Paris, 2001. MR 2003c:11144 Zbl 1031.11064

[Pollack 2003] R. Pollack, "On the $p$-adic $L$-function of a modular form at a supersingular prime", Duke Math. J. 118:3 (2003), 523-558. MR 2004e:11050 Zbl 1074.11061

[Schneider and Teitelbaum 2003] P. Schneider and J. Teitelbaum, "Algebras of p-adic distributions and admissible representations", Invent. Math. 153:1 (2003), 145-196. MR 2004g:22015 Zbl 1028.11070

[Sprung 2009] F. I. Sprung, "Iwasawa theory for elliptic curves at supersingular primes: beyond the case $a_{p}=0$ ”, preprint, 2009. arXiv 0903.3419v1

Communicated by Karl Rubin

Received 2010-11-26 Revised 2011-02-23 
antonio.lei@mcgill.ca

Current address:

d.a.loeffler@warwick.ac.uk

s.zerbes@exeter.ac.uk
School of Mathematical Sciences, Monash University, VIC 3800, Australia

Department of Mathematics and Statistics, Burnside Hall, McGill University, 805 Rue Sherbrooke Ouest, Montréal, QC, H3A OB9, Canada

Mathematics Institute, Zeeman Building,

University of Warwick, Coventry, CV4 7AL, United Kingdom

Mathematics Research Institute, Harrison Building,

University of Exeter, Exeter, EX4 4QF, United Kingdom 


\section{Algebra \& Number Theory}

msp.berkeley.edu/ant

\section{EDITORS}

MANAGING EDITOR

Bjorn Poonen

Massachusetts Institute of Technology

Cambridge, USA

\author{
EDITORIAL BOARD CHAIR \\ David Eisenbud \\ University of California \\ Berkeley, USA
}

\section{BOARD OF EDITORS}

Georgia Benkart

Dave Benson

Richard E. Borcherds

John H. Coates

J-L. Colliot-Thélène

Brian D. Conrad

Hélène Esnault

Hubert Flenner

Edward Frenkel

Andrew Granville

Joseph Gubeladze

Ehud Hrushovski

Craig Huneke

Mikhail Kapranov

Yujiro Kawamata

János Kollár

Yuri Manin

Barry Mazur

Philippe Michel

Susan Montgomery
University of Wisconsin, Madison, USA

University of Aberdeen, Scotland

University of California, Berkeley, USA

University of Cambridge, UK

CNRS, Université Paris-Sud, France

University of Michigan, USA

Universität Duisburg-Essen, Germany

Ruhr-Universität, Germany

University of California, Berkeley, USA

Université de Montréal, Canada

San Francisco State University, USA

Hebrew University, Israel

University of Kansas, USA

Yale University, USA

University of Tokyo, Japan

Princeton University, USA

Northwestern University, USA

Harvard University, USA

École Polytechnique Fédérale de Lausanne

University of Southern California, USA
Shigefumi Mori

Raman Parimala

Jonathan Pila

Victor Reiner

Karl Rubin

Peter Sarnak

Joseph H. Silverman

Michael Singer

Ronald Solomon

Vasudevan Srinivas

J. Toby Stafford

Bernd Sturmfels

Richard Taylor

Ravi Vakil

Michel van den Bergh

Marie-France Vignéras

Kei-Ichi Watanabe

Andrei Zelevinsky

Efim Zelmanov
RIMS, Kyoto University, Japan

Emory University, USA

University of Oxford, UK

University of Minnesota, USA

University of California, Irvine, USA

Princeton University, USA

Brown University, USA

North Carolina State University, USA

Ohio State University, USA

Tata Inst. of Fund. Research, India

University of Michigan, USA

University of California, Berkeley, USA

Harvard University, USA

Stanford University, USA

Hasselt University, Belgium

Université Paris VII, France

Nihon University, Japan

Northeastern University, USA

University of California, San Diego, USA

\section{PRODUCTION}

contact@msp.org

Silvio Levy, Scientific Editor

See inside back cover or www.jant.org for submission instructions.

The subscription price for 2011 is US \$150/year for the electronic version, and \$210/year (+\$35 shipping outside the US) for print and electronic. Subscriptions, requests for back issues from the last three years and changes of subscribers address should be sent to Mathematical Sciences Publishers, Department of Mathematics, University of California, Berkeley, CA 94720-3840, USA.

Algebra \& Number Theory (ISSN 1937-0652) at Mathematical Sciences Publishers, Department of Mathematics, University of California, Berkeley, CA 94720-3840 is published continuously online. Periodical rate postage paid at Berkeley, CA 94704, and additional mailing offices.

ANT peer review and production are managed by EditFLOW ${ }^{\circledR}$ from Mathematical Sciences Publishers.

PUBLISHED BY

mathematical sciences publishers

http://msp.org/

A NON-PROFIT CORPORATION

Typeset in IAT $_{\mathrm{E}} \mathrm{X}$

Copyright @2011 by Mathematical Sciences Publishers 


\section{Algebra \& Number Theory}

\section{Volume $5 \quad$ No. $8 \quad 2011$}

The behavior of Hecke $L$-functions of real quadratic fields at $s=0$ BYUNGHEUP JUN and JUNGYUN LEE

The Picard group of a $K 3$ surface and its reduction modulo $p$

ANDREAS-STEPHAN ELSENHANS and JÖRG JAHNEL

Linear determinantal equations for all projective schemes JESSICA SIDMAN and GREGORY G. SMITH

Involutions, weights and $p$-local structure

GEOFFREY R. ROBINSON

Parametrizing quartic algebras over an arbitrary base

MELANIE MatchetT WoOD

Coleman maps and the $p$-adic regulator

ANTONIO LEI, DAVID LOEFFLER and SARAH LIVIA ZERBES

Conjecture de Shafarevitch effective pour les revêtements cycliques 